\title{
Properties of the Exceptional $\left(X_{\ell}\right)$ Laguerre and Jacobi Polynomials
}

\author{
Choon-Lin $\mathrm{HO}^{\dagger}$, Satoru ODAKE ${ }^{\ddagger}$ and Ryu SASAKI ${ }^{\S}$ \\ $\dagger$ Department of Physics, Tamkang University, Tamsui 251, Taiwan (R.O.C.) \\ E-mail: hcl@mail.tku.edu.tw \\ $\ddagger$ Department of Physics, Shinshu University, Matsumoto 390-8621, Japan \\ E-mail: odake@azusa.shinshu-u.ac.jp \\ $\S$ Yukawa Institute for Theoretical Physics, Kyoto University, Kyoto 606-8502, Japan \\ E-mail:ryu@yukawa.kyoto-u.ac.jp
}

Received April 18, 2011, in final form November 19, 2011; Published online November 25, 2011

http://dx.doi.org/10.3842/SIGMA.2011.107

\begin{abstract}
We present various results on the properties of the four infinite sets of the exceptional $X_{\ell}$ polynomials discovered recently by Odake and Sasaki [Phys. Lett. B 679 (2009), 414-417; Phys. Lett. B 684 (2010), 173-176]. These $X_{\ell}$ polynomials are global solutions of second order Fuchsian differential equations with $\ell+3$ regular singularities and their confluent limits. We derive equivalent but much simpler looking forms of the $X_{\ell}$ polynomials. The other subjects discussed in detail are: factorisation of the Fuchsian differential operators, shape invariance, the forward and backward shift operations, invariant polynomial subspaces under the Fuchsian differential operators, the Gram-Schmidt orthonormalisation procedure, three term recurrence relations and the generating functions for the $X_{\ell}$ polynomials.
\end{abstract}

Key words: exceptional orthogonal polynomials; Gram-Schmidt process; Rodrigues formulas; generating functions

2010 Mathematics Subject Classification: 42C05; 33E30; 81Q05

\section{Introduction}

Four sets of infinitely many exceptional $\left(X_{\ell}\right)$ polynomials satisfying second order differential equations were introduced recently by two of the present authors $[1,2]$. They were obtained as the main part of the eigenfunctions of exactly solvable one-dimensional quantum mechanical systems which were deformations of the well-known solvable systems of the radial oscillator $[3,4]$ and the trigonometric Darboux-Pöschl-Teller (DPT) potential [5] by a degree $\ell$ eigenpolynomial. Thus the orthogonality and completeness of the $X_{\ell}$ polynomials are automatically guaranteed. These polynomials, termed exceptional Laguerre and Jacobi polynomials, have two types in each family, L1, L2 and J1 and J2. The Laguerre family L1 (L2) is obtained from the Jacobi family J1 (J2) by the well-known limit (E.28), which takes the Jacobi polynomials to the Laguerre polynomials. The J1 and J2 are mirror images of each other, see (E.14), but their limits L1 and L2 are clearly distinct. These polynomials are exceptional in the sense that they start at degree $\ell(\ell=1,2, \ldots)$ rather than degree 0 constant term. Thus they are not constrained by Bochner's theorem [6], which states that the orthogonal polynomials (starting with degree 0) satisfying a second order differential equations are very limited. Namely, they are only the classical orthogonal polynomials, the Hermite, Laguerre, Jacobi and Bessel polynomials.

The concept of exceptional orthogonal polynomials was introduced in 2008 by Gomez-Ullate et al. [7,8]. Within the Sturm-Liouville theory they constructed $X_{1}$ Laguerre and $X_{1}$ Ja- 
cobi polynomials, which turned out to be the first members of the infinite families. The results in $[7,8]$ were reformulated in the framework of quantum mechanics and shape-invariant potentials [9] by Quesne and collaborators [10, 11]. They found the first member of the deformed hyperbolic DPT potential family, which was also given in [1]. Quantum mechanical reformulation offers two merits. Firstly, the orthogonality and completeness of the obtained eigenfunctions are guaranteed. Secondly, the well established solution mechanism of shape invariance combined with Crum's method [12], or the so-called factorisation method [3] or the susy quantum mechanics [4] is available. For systems consisting of discrete eigenvalues only, shape invariance is a well-known sufficient condition for exact solvability of one-dimensional Schrödinger equation. The discovery of the four sets of infinitely many exceptional orthogonal polynomials was achieved by pursuing shape invariant deformation $[1,2]$. To be more precise, deformation of the potential by keeping the property of shape invariance. After the first paper on infinitely many $X_{\ell}$ polynomials [1], Quesne reported a type II $X_{2}$ Laguerre polynomials [13, 14], which led to the discovery of the L2 family of $X_{\ell}$ Laguerre polynomials [2]. In a previous paper [15], two of the present authors unveiled infinitely many polynomial identities of degree $3 \ell$ involving cubic products of the Laguerre or the Jacobi polynomials, which encode the information of exact solvability of the differential equations governing the $X_{\ell}$ polynomials.

In this paper we explore various properties of the $X_{\ell}$ polynomials. We emphasise that the J1 and J2 polynomials are the global solutions of a Fuchsian differential equation having $\ell+3$ regular singularities. They are located at $\pm 1, \infty$ and the $\ell$ zeros of the polynomial $\xi_{\ell}(\eta ; \boldsymbol{\lambda})(2.2),(2.4)$ which is used for the deformation. Factorisation and shape invariance are reformulated accordingly, leading to Rodrigues formulas and the forward and backward shift operations. The existence of the extra regular singularities implies that the ordinary vector space spanned by degree $n$ polynomials $\mathcal{V}_{0, n}=\operatorname{Span}\left[1, x, \ldots, x^{n}\right]$ is not invariant under the Fuchsian differential operator (6.2). Appropriate invariant polynomial subspaces are introduced and their properties are used to derive the explicit forms of the exceptional polynomials. Some of the important subjects in orthogonal polynomial theory, namely, the Gram-Schmidt orthonormalisation, the generating functions, three term recurrence relations, the zeros of these orthogonal polynomials, etc, are also discussed. New infinitely many polynomial identities underlying the forward and backward shift operations are also reported.

The plan of this paper is as follows. In Section 2 we present the explicit forms of the four sets of infinitely many exceptional orthogonal polynomials together with their weight functions and the normalisation constants. They are equal to those reported earlier [1, 2], but look much simpler than the original ones. The new forms of the polynomials reveal the structure of the theory. In Section 3 the Fuchsian differential equations governing these polynomials are discussed. Shape invariance and Rodrigues formulas are presented in Section 4. The identities underlying the forward and backward shift relations are presented in Section 5. The polynomial subspaces invariant under the Fuchsian differential operator are discussed in Section 6. This gives another concise proof of the new forms of the exceptional polynomials. In Section 7 we provide the integration formula which is essential for the Gram-Schmidt construction in Section 8. Section 9 gives the generating functions for the $X_{\ell}$ polynomials. The double generating function, that is, the generating function of the generating functions, is presented for the L1 and L2 exceptional Laguerre polynomials. A substitute of the three term recurrence relations for the $X_{\ell}$ polynomials is introduced in Section 10. In Section 11 we state the qualitative features of the extra zeros of the $X_{\ell}$ polynomials without proof. The final section is for a summary and comments. Some technical details are relegated to Appendices. The equality of the new and original forms of the $X_{\ell}$ polynomials is demonstrated in Appendix A. Forward and backward shift relations are proved in Appendix B. Derivation of the integration formula is provided in Appendix C. The properties of the $X_{\ell}$ Jacobi polynomials as the solutions of the quantum mechanical systems 
with deformed hyperbolic DPT potentials are summarised in Appendix D. A concise summary of some important properties of the Laguerre and Jacobi polynomials is given in Appendix E for self-containedness.

Throughout this paper we stick to the notation of our previous papers $[1,2,15,16]$. Reference to the quantum mechanical language is made minimal in order to attain wider readership than before. Most concepts and formulas are common to the four sets of exceptional polynomials. As far as possible we use generic formulas valid for all the four different sets of $X_{\ell}$ polynomials, in order to emphasise the underlying structure and at the same time to avoid redundancy.

\section{Exceptional Laguerre and Jacobi polynomials}

Here we present four sets of infinitely many exceptional orthogonal polynomials [1, 2], among them two are deformations of the Laguerre polynomials, and the others are deformations of the Jacobi polynomials. They are expressed as a bilinear form of the original polynomials, the Laguerre or Jacobi polynomials and the deforming polynomials, depending on the set of parameters $\boldsymbol{\lambda}$ and their shifts $\boldsymbol{\delta}$ and a non-negative integer $\ell$, which is the degree of the deforming polynomials. It is important to stress that these explicit forms (2.1)-(2.4) can be derived by the Darboux-Crum transformations starting from the original (Laguerre or Jacobi) polynomials $[17,18]$. The two sets of exceptional Laguerre polynomials $(\ell=0,1,2, \ldots, n=0,1,2, \ldots)$ are:

$$
P_{\ell, n}(\eta ; \boldsymbol{\lambda}) \stackrel{\text { def }}{=}\left\{\begin{array}{c}
\xi_{\ell}(\eta ; \boldsymbol{\lambda}+\boldsymbol{\delta}) P_{n}(\eta ; g+\ell-1)-\xi_{\ell}(\eta ; \boldsymbol{\lambda}) \partial_{\eta} P_{n}(\eta ; g+\ell-1) \\
\left(n+g+\frac{1}{2}\right)^{-1}\left(\left(g+\frac{1}{2}\right) \xi_{\ell}(\eta ; \boldsymbol{\lambda}+\boldsymbol{\delta}) P_{n}(\eta ; g+\ell+1)\right. \\
\left.+\eta \xi_{\ell}(\eta ; \boldsymbol{\lambda}) \partial_{\eta} P_{n}(\eta ; g+\ell+1)\right)
\end{array}\right.
$$

in which $\boldsymbol{\lambda} \stackrel{\text { def }}{=} g>0$ and $\boldsymbol{\delta} \stackrel{\text { def }}{=} 1$ and

$$
P_{n}(\eta ; g) \stackrel{\text { def }}{=} L_{n}^{\left(g-\frac{1}{2}\right)}(\eta), \quad \xi_{\ell}(\eta ; g) \stackrel{\text { def }}{=}\left\{\begin{array}{l}
L_{\ell}^{\left(g+\ell-\frac{3}{2}\right)}(-\eta), \\
L_{\ell}^{\left(-g-\ell-\frac{1}{2}\right)}(\eta) .
\end{array}\right.
$$

The two sets of exceptional Jacobi polynomials $(\ell=0,1,2, \ldots, n=0,1,2, \ldots)$ are $^{1}$ :

$$
P_{\ell, n}(\eta ; \boldsymbol{\lambda}) \stackrel{\text { def }}{=}\left\{\begin{array}{r}
\left(n+h+\frac{1}{2}\right)^{-1}\left(\left(h+\frac{1}{2}\right) \xi_{\ell}(\eta ; \boldsymbol{\lambda}+\boldsymbol{\delta}) P_{n}(\eta ; g+\ell-1, h+\ell+1)\right. \\
\left.+(1+\eta) \xi_{\ell}(\eta ; \boldsymbol{\lambda}) \partial_{\eta} P_{n}(\eta ; g+\ell-1, h+\ell+1)\right), \\
\left(n+g+\frac{1}{2}\right)^{-1}\left(\left(g+\frac{1}{2}\right) \xi_{\ell}(\eta ; \boldsymbol{\lambda}+\boldsymbol{\delta}) P_{n}(\eta ; g+\ell+1, h+\ell-1)\right. \\
\left.-(1-\eta) \xi_{\ell}(\eta ; \boldsymbol{\lambda}) \partial_{\eta} P_{n}(\eta ; g+\ell+1, h+\ell-1)\right),
\end{array}\right.
$$

in which $\boldsymbol{\lambda} \stackrel{\text { def }}{=}(g, h), g>0, h>0, \delta \stackrel{\text { def }}{=}(1,1)$ and

$$
\begin{aligned}
& P_{n}(\eta ; g, h) \stackrel{\text { def }}{=} P_{n}^{\left(g-\frac{1}{2}, h-\frac{1}{2}\right)}(\eta), \\
& \xi_{\ell}(\eta ; g, h) \stackrel{\text { def }}{=} \begin{cases}P_{\ell}^{\left(g+\ell-\frac{3}{2},-h-\ell-\frac{1}{2}\right)}(\eta), & g>h>0, \\
P_{\ell}^{\left(-g-\ell-\frac{1}{2}, h+\ell-\frac{3}{2}\right)}(\eta), & h>g>0 .\end{cases}
\end{aligned}
$$

\footnotetext{
${ }^{1}$ It should be remarked that the naming J1 and J2 are interchanged from the previous ones [2] in order to respect the logical consistency rather than hysteresis.
} 
It should be stressed that the deforming polynomial $\xi_{\ell}(\eta ; \boldsymbol{\lambda})$ does not have a zero in the domain of orthogonality, $(0, \infty)$ for L1 and L2 and $(-1,1)$ for J1 and J2. This is demonstrated explicitly in (2.39), (2.40) of [15]. These $X_{\ell}$ polynomials have the following general structure

$$
\begin{aligned}
d_{0}(n, \boldsymbol{\lambda}) P_{\ell, n}(\eta ; \boldsymbol{\lambda})= & d_{1}(\boldsymbol{\lambda}) \xi_{\ell}(\eta ; \boldsymbol{\lambda}+\boldsymbol{\delta}) P_{n}(\eta ; \boldsymbol{\lambda}+\ell \boldsymbol{\delta}+\tilde{\boldsymbol{\delta}}) \\
& -d_{2}(\eta) \xi_{\ell}(\eta ; \boldsymbol{\lambda}) \partial_{\eta} P_{n}(\eta ; \boldsymbol{\lambda}+\ell \boldsymbol{\delta}+\tilde{\boldsymbol{\delta}})
\end{aligned}
$$

where

$$
\begin{aligned}
& d_{0}(n, \boldsymbol{\lambda}) \stackrel{\text { def }}{=}\left\{\begin{array} { l l } 
{ 1 , } & { ( \mathrm { L } 1 ) } \\
{ n + g + \frac { 1 } { 2 } , } & { ( \mathrm { L } 2 , \mathrm { J } 2 ) } \\
{ n + h + \frac { 1 } { 2 } , } & { ( \mathrm { J } 1 ) }
\end{array} \quad \tilde { \boldsymbol { \delta } } \stackrel { \text { def } } { = } \left\{\begin{array}{l}
\mp 1, \\
\mp(1,-1),
\end{array}\right.\right. \\
& d_{1}(\boldsymbol{\lambda}) \stackrel{\text { def }}{=}\left\{\begin{array} { l l } 
{ 1 , } & { ( \mathrm { L } 1 ) } \\
{ g + \frac { 1 } { 2 } , } & { ( \mathrm { L } 2 , \mathrm { J } 2 ) } \\
{ h + \frac { 1 } { 2 } , } & { ( \mathrm { J } 1 ) }
\end{array} \quad d _ { 2 } ( \eta ) \stackrel { \text { def } } { = } \left\{\begin{array}{l}
1, \\
-\eta, \\
\mp(1 \pm \eta) .
\end{array}\right.\right.
\end{aligned}
$$

We introduce a linear map $\Xi_{\ell, \boldsymbol{\lambda}}[\cdot]$ for a differentiable function $p(\eta)$,

$$
\Xi_{\ell, \boldsymbol{\lambda}}[p(\eta)] \stackrel{\text { def }}{=} d_{1}(\boldsymbol{\lambda}) \xi_{\ell}(\eta ; \boldsymbol{\lambda}+\boldsymbol{\delta}) p(\eta)-d_{2}(\eta) \xi_{\ell}(\eta ; \boldsymbol{\lambda}) \partial_{\eta} p(\eta) .
$$

Then the $X_{\ell}$ polynomial (2.5) is expressed succinctly as

$$
d_{0}(n, \boldsymbol{\lambda}) P_{\ell, n}(\eta ; \boldsymbol{\lambda})=\Xi_{\ell, \boldsymbol{\lambda}}\left[P_{n}(\eta ; \boldsymbol{\lambda}+\ell \boldsymbol{\delta}+\tilde{\boldsymbol{\delta}})\right] .
$$

The $X_{0}$ polynomials $P_{0, n}(\eta ; \boldsymbol{\lambda})=P_{n}(\eta ; \boldsymbol{\lambda})$ are the undeformed polynomials, i.e., the Laguerre or the Jacobi polynomials themselves. Therefore the above formulas (2.1), (2.3) and (2.5) for $\ell=0$ are non-trivial identities among the Laguerre or the Jacobi polynomials

$$
d_{0}(n, \boldsymbol{\lambda}) P_{n}(\eta ; \boldsymbol{\lambda})=d_{1}(\boldsymbol{\lambda}) P_{n}(\eta ; \boldsymbol{\lambda}+\tilde{\boldsymbol{\delta}})-d_{2}(\eta) \partial_{\eta} P_{n}(\eta ; \boldsymbol{\lambda}+\tilde{\boldsymbol{\delta}}) .
$$

This is shown by using (E.2), (E.15) and the following; (E.11) for L1, (E.11) and (E.12) for L2, (E.22) for J1 and (E.25) for J2. The $X_{\ell}$ polynomials $P_{\ell, n}(\eta ; \boldsymbol{\lambda})$ are degree $\ell+n$ polynomials in $\eta$ and start at degree $\ell$ :

$$
P_{\ell, 0}(\eta ; \boldsymbol{\lambda})=\xi_{\ell}(\eta ; \boldsymbol{\lambda}+\boldsymbol{\delta})
$$

They are orthogonal with respect to the weight function $\mathcal{W}_{\ell}(\eta, \lambda)$ which is a deformation of the weight function $W(\eta ; \boldsymbol{\lambda})$ for the Laguerre or Jacobi polynomials:

$$
\begin{aligned}
& \int P_{\ell, n}(\eta ; \boldsymbol{\lambda}) P_{\ell, m}(\eta ; \boldsymbol{\lambda}) \mathcal{W}_{\ell}(\eta ; \boldsymbol{\lambda}) d \eta=h_{\ell, n}(\boldsymbol{\lambda}) \delta_{n m}, \\
& \mathcal{W}_{\ell}(\eta ; \boldsymbol{\lambda}) \stackrel{\text { def }}{=} \frac{W(\eta ; \boldsymbol{\lambda}+\ell \boldsymbol{\delta})}{\xi_{\ell}(\eta ; \boldsymbol{\lambda})^{2}}, \\
& W(\eta ; \boldsymbol{\lambda}) \stackrel{\text { def }}{=} \begin{cases}\frac{1}{2} e^{-\eta} \eta^{g-\frac{1}{2}}, & 0<\eta<\infty, \\
\frac{1}{2^{g+h+1}}(1-\eta)^{g-\frac{1}{2}}(1+\eta)^{h-\frac{1}{2}}, & -1<\eta<1 .\end{cases}
\end{aligned}
$$

The normalisation constants are meromorphic functions of the parameters $g, h$ and $\ell$ :

$$
(\mathrm{L}): \quad h_{n}(g) \stackrel{\text { def }}{=} \frac{1}{2 n !} \Gamma\left(n+g+\frac{1}{2}\right)
$$




$$
\begin{aligned}
& h_{\ell, n}(g) \stackrel{\text { def }}{=} h_{n}(g+\ell) \times \begin{cases}\frac{n+g+2 \ell-\frac{1}{2}}{n+g+\ell-\frac{1}{2}}, & (\mathrm{~L} 1) \\
\frac{n+g+\ell+\frac{1}{2}}{n+g+\frac{1}{2}}, & (\mathrm{~L} 2)\end{cases} \\
&(\mathrm{J}): \quad h_{n}(g, h) \stackrel{\text { def }}{=} \frac{\Gamma\left(n+g+\frac{1}{2}\right) \Gamma\left(n+h+\frac{1}{2}\right)}{2 n !(2 n+g+h) \Gamma(n+g+h)}, \\
& h_{\ell, n}(g, h) \stackrel{\text { def }}{=} h_{n}(g+\ell, h+\ell) \times\left\{\begin{array}{l}
\frac{\left(n+h+\ell+\frac{1}{2}\right)\left(n+g+2 \ell-\frac{1}{2}\right)}{\left(n+h+\frac{1}{2}\right)\left(n+g+\ell-\frac{1}{2}\right)} \\
\frac{\left(n+g+\ell+\frac{1}{2}\right)\left(n+h+2 \ell-\frac{1}{2}\right)}{\left(n+g+\frac{1}{2}\right)\left(n+h+\ell-\frac{1}{2}\right)} .
\end{array}\right.
\end{aligned}
$$

As stressed in $\S 3$ of [2], the J1 and J2 polynomials are the mirror images of each other, in the sense $\eta \leftrightarrow-\eta$ and $g \leftrightarrow h$, as exemplified by the relation $\xi_{\ell}^{\mathrm{J} 2}(\eta ; g, h)=(-1)^{\ell} \xi_{\ell}^{\mathrm{J} 1}(-\eta ; h, g)$. However, they lead to the two different sets of the exceptional Laguerre polynomials, J1 $\rightarrow \mathrm{L} 1$, $\mathrm{J} 2 \rightarrow \mathrm{L} 2$. In terms of the limit formulas

$$
\lim _{\beta \rightarrow \infty} P_{n}^{(\alpha, \pm \beta)}\left(1-\frac{2 x}{\beta}\right)=L_{n}^{(\alpha)}( \pm x),
$$

it is easy to see the relations $P_{\ell, n}(\eta ; \boldsymbol{\lambda})(2.3) \rightarrow(2.1)$ together with the deforming polynomials $\xi_{\ell}(\eta ; \boldsymbol{\lambda})(2.4) \rightarrow(2.2)$, the normalisation constants [(2.10) with $\left.(2.15)\right] \rightarrow[(2.10)$ with $(2.13)]$ and others. The explicit forms of the $X_{\ell}$ polynomials (2.1) and (2.3) are much simpler than those given in the previous papers $[1,2]$. In Appendix A we will give simple demonstration that these apparently different forms of $X_{\ell}$ polynomials are in fact equal.

The basic ingredients of the theory of exceptional orthogonal polynomials are the base polynomial $P_{n}$ and the deforming polynomial $\xi_{\ell}$. They satisfy the second order differential equations (E.4) and (E.17), which can be expressed as

$$
\begin{aligned}
& c_{2}(\eta) \partial_{\eta}^{2} P_{n}(\eta ; \boldsymbol{\lambda})+c_{1}(\eta, \boldsymbol{\lambda}) \partial_{\eta} P_{n}(\eta ; \boldsymbol{\lambda})=-\frac{1}{4} \mathcal{E}_{n}(\boldsymbol{\lambda}) P_{n}(\eta ; \boldsymbol{\lambda}), \\
& c_{2}(\eta) \partial_{\eta}^{2} \xi_{\ell}(\eta ; \boldsymbol{\lambda})+\tilde{c}_{1}(\eta, \boldsymbol{\lambda}, \ell) \partial_{\eta} \xi_{\ell}(\eta ; \boldsymbol{\lambda})=-\frac{1}{4} \widetilde{\mathcal{E}}_{\ell}(\boldsymbol{\lambda}) \xi_{\ell}(\eta ; \boldsymbol{\lambda}),
\end{aligned}
$$

where

$$
\begin{aligned}
& c_{1}(\eta, \boldsymbol{\lambda}) \stackrel{\text { def }}{=}\left\{\begin{array} { l l } 
{ g + \frac { 1 } { 2 } - \eta , } & { ( \mathrm { L } ) } \\
{ h - g - ( g + h + 1 ) \eta , } & { ( \mathrm { J } ) }
\end{array} \quad c _ { 2 } ( \eta ) \stackrel { \text { def } } { = } \left\{\begin{array}{l}
\eta, \\
1-\eta^{2},
\end{array}\right.\right. \\
& \tilde{c}_{1}(\eta, \boldsymbol{\lambda}, \ell) \stackrel{\text { def }}{=} \begin{cases} \pm\left(g+\ell-\frac{1}{2}+\eta\right), & (\mathrm{L} 1 / \mathrm{L} 2) \\
\mp(g+h+2 \ell-1+(g-h) \eta), & (\mathrm{J} 1 / \mathrm{J} 2)\end{cases} \\
& \mathcal{E}_{n}(\boldsymbol{\lambda}) \stackrel{\text { def }}{=}\left\{\begin{array} { l l l } 
{ 4 n , } & { ( \mathrm { L } ) } \\
{ 4 n ( n + g + h ) , } & { ( \mathrm { J } ) }
\end{array} \quad \widetilde { \mathcal { E } } _ { \ell } ( \boldsymbol { \lambda } ) \stackrel { \text { def } } { = } \left\{\begin{array}{ll}
\mp 4 \ell, & (\mathrm{L} 1 / \mathrm{L} 2) \\
4 \ell(\ell \pm g \mp h-1) . & (\mathrm{J} 1 / \mathrm{J} 2)
\end{array}\right.\right.
\end{aligned}
$$

The deforming polynomial $\xi_{\ell}(\eta ; \boldsymbol{\lambda}+\boldsymbol{\delta})$ is expressed in terms of $\xi_{\ell}(\eta ; \boldsymbol{\lambda})$,

$$
d_{1}(\boldsymbol{\lambda}) \xi_{\ell}(\eta ; \boldsymbol{\lambda}+\boldsymbol{\delta})=d_{1}(\boldsymbol{\lambda}+\ell \boldsymbol{\delta}) \xi_{\ell}(\eta ; \boldsymbol{\lambda})+d_{2}(\eta) \partial_{\eta} \xi_{\ell}(\eta ; \boldsymbol{\lambda})
$$

with $d_{1}$ and $d_{2}$ defined in (2.6). This is shown by (E.2), (E.15) and various identities of the polynomials; (E.11) for L1, (E.11) and (E.12) for L2, (E.22) for J1 and (E.25) for J2. Conversely $\xi_{\ell}(\eta ; \boldsymbol{\lambda})$ is expressed in terms of $\xi_{\ell}(\eta ; \boldsymbol{\lambda}+\boldsymbol{\delta})$,

$$
d_{3}(\boldsymbol{\lambda}+\ell \boldsymbol{\delta}, \ell) \xi_{\ell}(\eta ; \boldsymbol{\lambda})=d_{3}(\boldsymbol{\lambda}, \ell) \xi_{\ell}(\eta ; \boldsymbol{\lambda}+\boldsymbol{\delta})+\frac{c_{2}(\eta)}{d_{2}(\eta)} \partial_{\eta} \xi_{\ell}(\eta ; \boldsymbol{\lambda}+\boldsymbol{\delta}),
$$


where

$$
d_{3}(\boldsymbol{\lambda}, \ell) \stackrel{\text { def }}{=} \begin{cases}g+\ell-\frac{1}{2}, & (\mathrm{~L} 1, \mathrm{~J} 1) \\ 1, & (\mathrm{~L} 2) \\ h+\ell-\frac{1}{2} . & (\mathrm{J} 2)\end{cases}
$$

This is shown in similar ways as above. The Laguerre and Jacobi differential equations for $P_{n}$ (2.17) can be factorised into the forward and backward shift relations for $P_{n}$ :

$$
\begin{aligned}
& c_{\mathcal{F}} \partial_{\eta} P_{n}(\eta ; \boldsymbol{\lambda})=f_{n}(\boldsymbol{\lambda}) P_{n-1}(\eta ; \boldsymbol{\lambda}+\boldsymbol{\delta}), \\
& c_{1}(\eta, \boldsymbol{\lambda}) P_{n-1}(\eta ; \boldsymbol{\lambda}+\boldsymbol{\delta})+c_{2}(\eta) \partial_{\eta} P_{n-1}(\eta ; \boldsymbol{\lambda}+\boldsymbol{\delta})=-\frac{1}{4} c_{\mathcal{F}} b_{n-1}(\boldsymbol{\lambda}) P_{n}(\eta ; \boldsymbol{\lambda}),
\end{aligned}
$$

where

$$
c_{\mathcal{F}} \stackrel{\text { def }}{=}\left\{\begin{array}{ll}
2, & (\mathrm{~L}) \\
-4, & (\mathrm{~J})
\end{array} \quad f_{n}(\boldsymbol{\lambda})=\left\{\begin{array}{ll}
-2, & (\mathrm{~L}) \\
-2(n+g+h), & (\mathrm{J})
\end{array} \quad b_{n-1}(\boldsymbol{\lambda})=-2 n .\right.\right.
$$

See (E.2), (E.3) and (E.15), (E.16) for the explicit forms of the forward and backward shift relations. Note that $f_{n}(\boldsymbol{\lambda})$ and $b_{n-1}(\boldsymbol{\lambda})$ are the factors of the eigenvalue

$$
\mathcal{E}_{n}(\boldsymbol{\lambda})=f_{n}(\boldsymbol{\lambda}) b_{n-1}(\boldsymbol{\lambda}), \quad n=0,1, \ldots
$$

\section{Fuchsian differential equations with extra $\ell$ regular singularities}

The exceptional Laguerre and Jacobi polynomials satisfy a second order linear differential equation in the entire complex $\eta$ plane:

$$
\widetilde{\mathcal{H}}_{\ell}(\boldsymbol{\lambda}) P_{\ell, n}(\eta ; \boldsymbol{\lambda})=\mathcal{E}_{\ell, n}(\boldsymbol{\lambda}) P_{\ell, n}(\eta ; \boldsymbol{\lambda}), \quad \mathcal{E}_{\ell, n}(\boldsymbol{\lambda})=\mathcal{E}_{n}(\boldsymbol{\lambda}+\ell \boldsymbol{\delta})
$$

in which the eigenvalue $\mathcal{E}_{n}$ is defined in (2.19). The $X_{\ell}$ polynomials are not constrained by Bochner's theorem [6] by the very fact that they start at degree $\ell$ (2.9) instead of degree 0 constant term. As with the Laguerre and Jacobi differential equations (2.17), the second order differential operator $\widetilde{\mathcal{H}}_{\ell}(\boldsymbol{\lambda})$ is factorised into the product of the forward shift operator $\mathcal{F}_{\ell}(\boldsymbol{\lambda})$ and the backward shift operator $\mathcal{B}_{\ell}(\boldsymbol{\lambda})$ :

$$
\begin{aligned}
& \widetilde{\mathcal{H}}_{\ell}(\boldsymbol{\lambda}) \stackrel{\text { def }}{=} \mathcal{B}_{\ell}(\boldsymbol{\lambda}) \mathcal{F}_{\ell}(\boldsymbol{\lambda}), \\
& \mathcal{F}_{\ell}(\boldsymbol{\lambda}) \stackrel{\text { def }}{=} c_{\mathcal{F}} \frac{\xi_{\ell}(\eta ; \boldsymbol{\lambda}+\boldsymbol{\delta})}{\xi_{\ell}(\eta ; \boldsymbol{\lambda})}\left(\frac{d}{d \eta}-\partial_{\eta} \log \xi_{\ell}(\eta ; \boldsymbol{\lambda}+\boldsymbol{\delta})\right), \\
& \mathcal{B}_{\ell}(\boldsymbol{\lambda}) \stackrel{\text { def }}{=}-4 c_{\mathcal{F}}^{-1} c_{2}(\eta) \frac{\xi_{\ell}(\eta ; \boldsymbol{\lambda})}{\xi_{\ell}(\eta ; \boldsymbol{\lambda}+\boldsymbol{\delta})}\left(\frac{d}{d \eta}+\frac{c_{1}(\eta, \boldsymbol{\lambda}+\ell \boldsymbol{\delta})}{c_{2}(\eta)}-\partial_{\eta} \log \xi_{\ell}(\eta ; \boldsymbol{\lambda})\right) .
\end{aligned}
$$

The differential operator $\widetilde{\mathcal{H}}_{\ell}(\boldsymbol{\lambda})$ is obtained from the factorised quantum mechanical Hamilto$\operatorname{nian}(16)$ of [1] and (3) of [2], by similarity transformation. Note that $\frac{c_{1}(\eta, \boldsymbol{\lambda}+\ell \boldsymbol{\delta})}{c_{2}(\eta)}=\partial_{\eta} \log W(\eta ; \boldsymbol{\lambda}+$ $(\ell+1) \boldsymbol{\delta})$. It is straightforward to derive the explicit form of $\widetilde{\mathcal{H}}_{\ell}(\boldsymbol{\lambda})$ :

$$
\begin{gathered}
\widetilde{\mathcal{H}}_{\ell}(\boldsymbol{\lambda})=-4\left(c_{2}(\eta) \frac{d^{2}}{d \eta^{2}}+\left(c_{1}(\eta, \boldsymbol{\lambda}+\ell \boldsymbol{\delta})-2 c_{2}(\eta) \partial_{\eta} \log \xi_{\ell}(\eta ; \boldsymbol{\lambda})\right) \frac{d}{d \eta}\right. \\
\left.+2 d_{1}(\boldsymbol{\lambda}) \frac{c_{2}(\eta)}{d_{2}(\eta)} \frac{\partial_{\eta} \xi_{\ell}(\eta ; \boldsymbol{\lambda}+\boldsymbol{\delta})}{\xi_{\ell}(\eta ; \boldsymbol{\lambda})}+\frac{1}{4} \widetilde{\mathcal{E}}_{\ell}(\boldsymbol{\lambda}+\boldsymbol{\delta})\right) .
\end{gathered}
$$


Use is made of the second order differential equations for $\xi_{\ell}(\eta ; \boldsymbol{\lambda}+\boldsymbol{\delta})(2.18)$ and the identity $(2.20)$ to derive the above simple result.

For $\ell=0$ the above differential equation (3.1) with (3.5) reduces to the second order differential equation for the Laguerre or Jacobi polynomials:

$$
\begin{array}{ll} 
& \widetilde{\mathcal{H}}_{0}(\boldsymbol{\lambda})=-4\left(c_{2}(\eta) \frac{d^{2}}{d \eta^{2}}+c_{1}(\eta, \boldsymbol{\lambda}) \frac{d}{d \eta}\right), \\
(\mathrm{L}): \quad & \widetilde{\mathcal{H}}_{0}(\boldsymbol{\lambda})=-4\left(\eta \frac{d^{2}}{d \eta^{2}}+\left(g+\frac{1}{2}-\eta\right) \frac{d}{d \eta}\right), \\
& \eta \partial_{\eta}^{2} P_{n}(\eta ; g)+\left(g+\frac{1}{2}-\eta\right) \partial_{\eta} P_{n}(\eta ; g)+n P_{n}(\eta ; g)=0, \\
(\mathrm{~J}): \quad & \widetilde{\mathcal{H}}_{0}(\boldsymbol{\lambda})=-4\left(\left(1-\eta^{2}\right) \frac{d^{2}}{d \eta^{2}}+(h-g-(g+h+1) \eta) \frac{d}{d \eta}\right), \\
& \left(1-\eta^{2}\right) \partial_{\eta}^{2} P_{n}(\eta ; g, h)+(h-g-(g+h+1) \eta) \partial_{\eta} P_{n}(\eta ; g, h) \\
& +n(n+g+h) P_{n}(\eta ; g, h)=0,
\end{array}
$$

which has, as is well-known, one regular singularity at $\eta=0$ and one irregular singularity at $\eta=\infty$ for the Laguerre case and three regular singularities at $\eta= \pm 1, \infty$ for the Jacobi case. For a non-negative integer $\ell$, the singularity structure of the second order differential equation (3.1) is again quite simple. It has extra regular singularities at the $\ell$ zeros of the deforming polynomial $\xi_{\ell}(\eta ; \boldsymbol{\lambda})$ :

$$
\eta=\eta_{j}, \quad \xi_{\ell}\left(\eta_{j} ; \boldsymbol{\lambda}\right)=0, \quad j=1,2, \ldots, \ell,
$$

and the corresponding exponents are the same for all the singular points:

$$
\text { \{exponents at } \left.\eta_{j}\right\}=\{0,3\}, \quad j=1,2, \ldots, \ell \text {. }
$$

In other words, the alternative solution of the second order linear differential equation (3.1) has a cubic zero at $\eta=\eta_{j}$. It is singular at $\eta=0, \infty$ for the L1 and L2 and at $\eta= \pm 1$ for the J1 and J2 with the exponents replaced by $g \rightarrow g+\ell, h \rightarrow h+\ell$. To the best of our knowledge, the two sets of $X_{\ell}$ orthogonal polynomials, J1 and J2 are the first examples of global solutions of Fuchsian differential equations having as many as $\ell+3$ regular singularities and forming a complete orthogonal system. The L1 and L2 are confluent types obtained from J1 and J2 by certain limits (E.28). By the way, it is elementary to show that $\xi_{\ell}(\eta ; \boldsymbol{\lambda})$ has only simple zeros.

Let us write down the explicit form of the above differential equation for the four cases:

$$
\begin{aligned}
(\mathrm{L} 1): \quad \eta \partial_{\eta}^{2} P_{\ell, n}(\eta ; \boldsymbol{\lambda}) & +\left(g+\ell+\frac{1}{2}-\eta-2 \frac{\eta \partial_{\eta} \xi_{\ell}(\eta ; \boldsymbol{\lambda})}{\xi_{\ell}(\eta ; \boldsymbol{\lambda})}\right) \partial_{\eta} P_{\ell, n}(\eta ; \boldsymbol{\lambda}) \\
& +\left(2 \frac{\eta \partial_{\eta} \xi_{\ell}(\eta ; \boldsymbol{\lambda}+\boldsymbol{\delta})}{\xi_{\ell}(\eta ; \boldsymbol{\lambda})}+n-\ell\right) P_{\ell, n}(\eta ; \boldsymbol{\lambda})=0 \\
(\mathrm{~L} 2): \quad \eta \partial_{\eta}^{2} P_{\ell, n}(\eta ; \boldsymbol{\lambda}) & +\left(g+\ell+\frac{1}{2}-\eta-2 \frac{\eta \partial_{\eta} \xi_{\ell}(\eta ; \boldsymbol{\lambda})}{\xi_{\ell}(\eta ; \boldsymbol{\lambda})}\right) \partial_{\eta} P_{\ell, n}(\eta ; \boldsymbol{\lambda}) \\
& +\left(-2 \frac{\left(g+\frac{1}{2}\right) \partial_{\eta} \xi_{\ell}(\eta ; \boldsymbol{\lambda}+\boldsymbol{\delta})}{\xi_{\ell}(\eta ; \boldsymbol{\lambda})}+n+\ell\right) P_{\ell, n}(\eta ; \boldsymbol{\lambda})=0, \\
(\mathrm{~J} 1): \quad\left(1-\eta^{2}\right) \partial_{\eta}^{2} P_{\ell, n}(\eta ; \boldsymbol{\lambda}) & \left.(g+h+2 \ell+1) \eta-2 \frac{\left(1-\eta^{2}\right) \partial_{\eta} \xi_{\ell}(\eta ; \boldsymbol{\lambda})}{\xi_{\ell}(\eta ; \boldsymbol{\lambda})}\right) \partial_{\eta} P_{\ell, n}(\eta ; \boldsymbol{\lambda})
\end{aligned}
$$




$$
\begin{aligned}
& +\left(-\frac{2\left(h+\frac{1}{2}\right)(1-\eta) \partial_{\eta} \xi_{\ell}(\eta ; \boldsymbol{\lambda}+\boldsymbol{\delta})}{\xi_{\ell}(\eta ; \boldsymbol{\lambda})}+\ell(\ell+g-h-1)+n(n+g+h+2 \ell)\right) \\
& \times P_{\ell, n}(\eta ; \boldsymbol{\lambda})=0, \\
(\mathrm{~J} 2): \quad\left(1-\eta^{2}\right) \partial_{\eta}^{2} P_{\ell, n}(\eta ; \boldsymbol{\lambda}) & +\left(h-g-(g+h+2 \ell+1) \eta-2 \frac{\left(1-\eta^{2}\right) \partial_{\eta} \xi_{\ell}(\eta ; \boldsymbol{\lambda})}{\xi_{\ell}(\eta ; \boldsymbol{\lambda})}\right) \partial_{\eta} P_{\ell, n}(\eta ; \boldsymbol{\lambda}) \\
& +\left(\frac{2\left(g+\frac{1}{2}\right)(1+\eta) \partial_{\eta} \xi_{\ell}(\eta ; \boldsymbol{\lambda}+\boldsymbol{\delta})}{\xi_{\ell}(\eta ; \boldsymbol{\lambda})}+\ell(\ell+h-g-1)+n(n+g+h+2 \ell)\right) \\
& \times P_{\ell, n}(\eta ; \boldsymbol{\lambda})=0 .
\end{aligned}
$$

Let us remark that the zeros of the shifted deforming polynomial $\xi_{\ell}(\eta ; \boldsymbol{\lambda}+\boldsymbol{\delta})$ are regular points. It is straightforward to verify by direct calculation that the L1, L2, J1 and J2 $X_{\ell}$ polynomials (2.1)-(2.4) for lower $\ell$ and $n$ really satisfy the above differential equations (3.9)-(3.12). For analytical proof see the subsequent sections.

\section{Shape invariance}

This section is a reformulation of the shape invariance in the language of ordinary differential equations. In one-dimensional quantum mechanics, shape invariance is a sufficient condition [9] for exact solvability and it was the guiding principle for the discovery of these $X_{\ell}$ orthogonal polynomials $[1,16,15,2]$. Let us introduce another second order linear differential operator $\widetilde{\mathcal{H}}_{\ell}^{(1)}(\boldsymbol{\lambda})$ by interchanging the order of the two factors $\mathcal{F}_{\ell}(\boldsymbol{\lambda})$ and $\mathcal{B}_{\ell}(\boldsymbol{\lambda})$ of $\widetilde{\mathcal{H}}_{\ell}(\boldsymbol{\lambda})(3.2)$ :

$$
\widetilde{\mathcal{H}}_{\ell}^{(1)}(\boldsymbol{\lambda}) \stackrel{\text { def }}{=} \mathcal{F}_{\ell}(\boldsymbol{\lambda}) \mathcal{B}_{\ell}(\boldsymbol{\lambda})
$$

It is obvious that these two operators are intertwined by $\mathcal{F}_{\ell}(\boldsymbol{\lambda})$ and $\mathcal{B}_{\ell}(\boldsymbol{\lambda})$ :

$$
\begin{aligned}
& \mathcal{F}_{\ell}(\boldsymbol{\lambda}) \mathcal{B}_{\ell}(\boldsymbol{\lambda}) \mathcal{F}_{\ell}(\boldsymbol{\lambda})=\mathcal{F}_{\ell}(\boldsymbol{\lambda}) \tilde{\mathcal{H}}_{\ell}(\boldsymbol{\lambda})=\widetilde{\mathcal{H}}_{\ell}^{(1)}(\boldsymbol{\lambda}) \mathcal{F}_{\ell}(\boldsymbol{\lambda}), \\
& \mathcal{B}_{\ell}(\boldsymbol{\lambda}) \mathcal{F}_{\ell}(\boldsymbol{\lambda}) \mathcal{B}_{\ell}(\boldsymbol{\lambda})=\widetilde{\mathcal{H}}_{\ell}(\boldsymbol{\lambda}) \mathcal{B}_{\ell}(\boldsymbol{\lambda})=\mathcal{B}_{\ell}(\boldsymbol{\lambda}) \widetilde{\mathcal{H}}_{\ell}^{(1)}(\boldsymbol{\lambda})
\end{aligned}
$$

which implies that these two associated linear differential operators $\widetilde{\mathcal{H}}_{\ell}(\boldsymbol{\lambda})$ and $\widetilde{\mathcal{H}}_{\ell}^{(1)}(\boldsymbol{\lambda})$ are iso-spectral except for the lowest eigenfunction $P_{\ell, 0}(\eta ; \boldsymbol{\lambda})(2.9)$ which is annihilated by $\mathcal{F}_{\ell}(\boldsymbol{\lambda})$, see (3.3):

$$
\mathcal{F}_{\ell}(\boldsymbol{\lambda}) \xi_{\ell}(\eta ; \boldsymbol{\lambda}+\boldsymbol{\delta})=0 \quad\left(\Rightarrow \widetilde{\mathcal{H}}_{\ell}(\boldsymbol{\lambda}) P_{\ell, 0}(\eta ; \boldsymbol{\lambda})=0\right)
$$

If we denote the set of eigenfunctions of $\widetilde{\mathcal{H}}_{\ell}^{(1)}(\boldsymbol{\lambda})$ as $\left\{P_{\ell, n}^{(1)}(\eta ; \boldsymbol{\lambda})\right\}(n=0,1, \ldots)$, with arbitrary normalisation, we obtain one to one correspondence of $\left\{P_{\ell, n}(\eta ; \boldsymbol{\lambda})\right\}$ and $\left\{P_{\ell, n}^{(1)}(\eta ; \boldsymbol{\lambda})\right\}$ except for the lowest eigenfunction of $\widetilde{\mathcal{H}}_{\ell}(\boldsymbol{\lambda})$ :

$$
\begin{aligned}
& \widetilde{\mathcal{H}}_{\ell}(\boldsymbol{\lambda}) P_{\ell, n}(\eta ; \boldsymbol{\lambda})=\mathcal{E}_{\ell, n}(\boldsymbol{\lambda}) P_{\ell, n}(\eta ; \boldsymbol{\lambda}), \quad n=0,1, \ldots, \\
& \widetilde{\mathcal{H}}_{\ell}^{(1)}(\boldsymbol{\lambda}) P_{\ell, n-1}^{(1)}(\eta ; \boldsymbol{\lambda})=\mathcal{E}_{\ell, n}(\boldsymbol{\lambda}) P_{\ell, n-1}^{(1)}(\eta ; \boldsymbol{\lambda}), \quad n=1,2, \ldots, \\
& \mathcal{F}_{\ell}(\boldsymbol{\lambda}) P_{\ell, n}(\eta ; \boldsymbol{\lambda}) \propto P_{\ell, n-1}^{(1)}(\eta ; \boldsymbol{\lambda}), \quad \mathcal{B}_{\ell}(\boldsymbol{\lambda}) P_{\ell, n-1}^{(1)}(\eta ; \boldsymbol{\lambda}) \propto P_{\ell, n}(\eta ; \boldsymbol{\lambda}) .
\end{aligned}
$$

This much is a trivial consequence of the factorisation of $\widetilde{\mathcal{H}}_{\ell}(\boldsymbol{\lambda})(3.2)$. The essential property of the two associated differential operators $\widetilde{\mathcal{H}}_{\ell}(\boldsymbol{\lambda})$ and $\widetilde{\mathcal{H}}_{\ell}^{(1)}(\boldsymbol{\lambda})$ is that $\widetilde{\mathcal{H}}_{\ell}^{(1)}(\boldsymbol{\lambda})$ has the same shape 
as $\widetilde{\mathcal{H}}_{\ell}(\boldsymbol{\lambda})$ with shifted parameters, $\boldsymbol{\lambda} \rightarrow \boldsymbol{\lambda}+\boldsymbol{\delta}$, and an additive constant corresponding to the lowest eigenvalue measured from the bottom:

$$
\begin{aligned}
& \widetilde{\mathcal{H}}_{\ell}^{(1)}(\boldsymbol{\lambda})=\widetilde{\mathcal{H}}_{\ell}(\boldsymbol{\lambda}+\boldsymbol{\delta})+\mathcal{E}_{1}(\boldsymbol{\lambda}+\ell \boldsymbol{\delta}), \quad \text { or } \\
& \mathcal{F}_{\ell}(\boldsymbol{\lambda}) \mathcal{B}_{\ell}(\boldsymbol{\lambda})=\mathcal{B}_{\ell}(\boldsymbol{\lambda}+\boldsymbol{\delta}) \mathcal{F}_{\ell}(\boldsymbol{\lambda}+\boldsymbol{\delta})+\mathcal{E}_{1}(\boldsymbol{\lambda}+\ell \boldsymbol{\delta}) .
\end{aligned}
$$

The system is called shape invariant if the above condition is satisfied. See $[1,2,15]$ for the definition of shape invariance within the framework of quantum mechanics. The above is an equivalent definition within the framework of the Sturm-Liouville theory.

Shape invariance allows us to choose the normalisation of $\left\{P_{\ell, n}^{(1)}(\eta ; \boldsymbol{\lambda})\right\}$ to achieve

$$
P_{\ell, n}^{(1)}(\eta ; \boldsymbol{\lambda})=P_{\ell, n}(\eta ; \boldsymbol{\lambda}+\boldsymbol{\delta}), \quad n=0,1, \ldots
$$

Then the above two relations (4.1), the forward and backward shift relations, give the constraints on the functional forms of $\left\{P_{\ell, n}(\eta ; \boldsymbol{\lambda})\right\}$ :

$$
\begin{aligned}
& \mathcal{F}_{\ell}(\boldsymbol{\lambda}) P_{\ell, n}(\eta ; \boldsymbol{\lambda})=f_{n}(\boldsymbol{\lambda}+\ell \boldsymbol{\delta}) P_{\ell, n-1}(\eta ; \boldsymbol{\lambda}+\boldsymbol{\delta}), \quad n=0,1, \ldots, \\
& \mathcal{B}_{\ell}(\boldsymbol{\lambda}) P_{\ell, n-1}(\eta ; \boldsymbol{\lambda}+\boldsymbol{\delta})=b_{n-1}(\boldsymbol{\lambda}+\ell \boldsymbol{\delta}) P_{\ell, n}(\eta ; \boldsymbol{\lambda}), \quad n=1,2, \ldots,
\end{aligned}
$$

where $f_{n}(\boldsymbol{\lambda})$ and $b_{n-1}(\boldsymbol{\lambda})$ are given in (2.25). These amount to a version of Rodrigues formula expressing $P_{\ell, n}(\eta ; \boldsymbol{\lambda})$ in terms of repeated application of the backward shift operators on the lowest degree eigenpolynomials with the $n$-th shifted parameters, $P_{\ell, 0}(\eta ; \boldsymbol{\lambda}+n \boldsymbol{\delta})=\xi_{\ell}(\eta ; \boldsymbol{\lambda}+$ $(n+1) \boldsymbol{\delta})$ :

$$
P_{\ell, n}(\eta ; \boldsymbol{\lambda})=\prod_{k=0}^{n-1} \frac{\mathcal{B}_{\ell}(\boldsymbol{\lambda}+k \boldsymbol{\delta})}{b_{n-k-1}(\boldsymbol{\lambda}+(\ell+k) \boldsymbol{\delta})} \cdot \xi_{\ell}(\eta ; \boldsymbol{\lambda}+(n+1) \boldsymbol{\delta}),
$$

where $\prod_{k=0}^{n-1} a_{k}=a_{0} a_{1} \cdots a_{n-1}$. For the $X_{\ell}$ Laguerre and Jacobi polynomials, the Rodrigues formula reads explicitly:

$$
\begin{aligned}
(\mathrm{L}): \quad P_{\ell, n}(\eta ; \boldsymbol{\lambda})= & \frac{1}{n !} \frac{\xi_{\ell}(\eta ; \boldsymbol{\lambda})}{e^{-\eta} \eta^{g+\ell-\frac{1}{2}}} \prod_{k=0}^{n-1}\left(\frac{d}{d \eta}+\partial_{\eta} \log \frac{\xi_{\ell}(\eta ; \boldsymbol{\lambda}+(k+1) \boldsymbol{\delta})}{\xi_{\ell}(\eta ; \boldsymbol{\lambda}+k \boldsymbol{\delta})}\right) \\
& \times \frac{e^{-\eta} \eta^{n+g+\ell-\frac{1}{2}}}{\xi_{\ell}(\eta ; \boldsymbol{\lambda}+n \boldsymbol{\delta})} \xi_{\ell}(\eta ; \boldsymbol{\lambda}+(n+1) \boldsymbol{\delta}), \\
(\mathrm{J}): \quad P_{\ell, n}(\eta ; \boldsymbol{\lambda})= & \frac{(-1)^{n}}{2^{n} n !} \frac{\xi_{\ell}(\eta ; \boldsymbol{\lambda})}{(1-\eta)^{g+\ell-\frac{1}{2}}(1+\eta)^{h+\ell-\frac{1}{2}}} \prod_{k=0}^{n-1}\left(\frac{d}{d \eta}+\partial_{\eta} \log \frac{\xi_{\ell}(\eta ; \boldsymbol{\lambda}+(k+1) \boldsymbol{\delta})}{\xi_{\ell}(\eta ; \boldsymbol{\lambda}+k \boldsymbol{\delta})}\right) \\
& \times \frac{(1-\eta)^{n+g+\ell-\frac{1}{2}}(1+\eta)^{n+h+\ell-\frac{1}{2}}}{\xi_{\ell}(\eta ; \boldsymbol{\lambda}+n \boldsymbol{\delta})} \xi_{\ell}(\eta ; \boldsymbol{\lambda}+(n+1) \boldsymbol{\delta}) .
\end{aligned}
$$

For $\ell=0, \xi_{0}(\eta ; \boldsymbol{\lambda})=1$, the above two formulas reduce to the well-known Rodrigues formulas for the Laguerre and Jacobi polynomials, (E.6) and (E.19). For $\ell=1$ these two formulas are equivalent to the Rodrigues-type formulas (77) and (52) in Gomez-Ullate et al. [7]. For lower $\ell$, it is straightforward to verify the shape invariance relation (4.2) by direct calculation. In [15] it was shown that the shape invariance relation is attributed to a new polynomial identity of degree $3 \ell$ involving cubic products of the Laguerre or Jacobi polynomials. These identities are proved elementarily by combining simple identities in [15].

It is a good challenge to derive more explicit expressions of the Rodrigues formulas for each of the four families of the exceptional orthogonal polynomials. 


\section{$5 \quad$ Forward and backward shift relations}

Again it is straightforward to verify that the above Rodrigues formulas provide the explicit forms of the four families of the $X_{\ell}$ polynomials (2.1)-(2.4) for lower $\ell$ and $n$ by direct calculation. For analytic proof, one needs to verify that the above forward (4.3) and backward (4.4) shift relations are actually satisfied by the four families of the $X_{\ell}$ polynomials (2.1)-(2.4). The forward and backward shift relations are attributed to new polynomial identities of degree $2 \ell+n-1$ and $2 \ell+n$ involving cubic products of the Laguerre or Jacobi polynomials in a similar way to the shape invariance relations [15]. The forward (F) (4.3) and backward (B) (4.4) shift relations read explicitly

$$
\begin{aligned}
(\mathrm{F}): \quad 0= & c_{\mathcal{F}}\left(\xi_{\ell}(\eta ; \boldsymbol{\lambda}+\boldsymbol{\delta}) \frac{d}{d \eta}-\partial_{\eta} \xi_{\ell}(\eta ; \boldsymbol{\lambda}+\boldsymbol{\delta})\right) P_{\ell, n}(\eta ; \boldsymbol{\lambda}) \\
& -f_{n}(\boldsymbol{\lambda}+\ell \boldsymbol{\delta}) \xi_{\ell}(\eta ; \boldsymbol{\lambda}) P_{\ell, n-1}(\eta ; \boldsymbol{\lambda}+\boldsymbol{\delta}), \\
(\mathrm{B}): \quad 0= & \left(\xi_{\ell}(\eta ; \boldsymbol{\lambda})\left(c_{2}(\eta) \frac{d}{d \eta}+c_{1}(\eta, \boldsymbol{\lambda}+\ell \boldsymbol{\delta})\right)-c_{2}(\eta) \partial_{\eta} \xi_{\ell}(\eta ; \boldsymbol{\lambda})\right) P_{\ell, n-1}(\eta ; \boldsymbol{\lambda}+\boldsymbol{\delta}) \\
& +\frac{1}{4} c_{\mathcal{F}} b_{n-1}(\boldsymbol{\lambda}+\ell \boldsymbol{\delta}) \xi_{\ell}(\eta ; \boldsymbol{\lambda}+\boldsymbol{\delta}) P_{\ell, n}(\eta ; \boldsymbol{\lambda}),
\end{aligned}
$$

where $P_{\ell, n}$ is given in (2.5).

The new polynomial identities are as follows:

(L1): $\left(\alpha=g+\ell-\frac{1}{2}\right)$

$$
\begin{aligned}
(\mathrm{F}): 0= & \left(L_{\ell}^{(\alpha)}(-x) \frac{d}{d x}-\partial_{x} L_{\ell}^{(\alpha)}(-x)\right)\left(L_{\ell}^{(\alpha)}(-x) L_{n}^{(\alpha-1)}(x)-L_{\ell}^{(\alpha-1)}(-x) \partial_{x} L_{n}^{(\alpha-1)}(x)\right) \\
& +L_{\ell}^{(\alpha-1)}(-x)\left(L_{\ell}^{(\alpha+1)}(-x) L_{n-1}^{(\alpha)}(x)-L_{\ell}^{(\alpha)}(-x) \partial_{x} L_{n-1}^{(\alpha)}(x)\right), \\
(\mathrm{B}): \quad 0= & \left(L_{\ell}^{(\alpha-1)}(-x)\left(x \frac{d}{d x}+\alpha+1-x\right)-x \partial_{x} L_{\ell}^{(\alpha-1)}(-x)\right) \\
& \times\left(L_{\ell}^{(\alpha+1)}(-x) L_{n-1}^{(\alpha)}(x)-L_{\ell}^{(\alpha)}(-x) \partial_{x} L_{n-1}^{(\alpha)}(x)\right) \\
& -n L_{\ell}^{(\alpha)}(-x)\left(L_{\ell}^{(\alpha)}(-x) L_{n}^{(\alpha-1)}(x)-L_{\ell}^{(\alpha-1)}(-x) \partial_{x} L_{n}^{(\alpha-1)}(x)\right),
\end{aligned}
$$

(L2): $\left(\alpha=g+\ell-\frac{1}{2}\right)$

$$
\begin{aligned}
(\mathrm{F}): 0= & \left(L_{\ell}^{(-\alpha-2)}(x) \frac{d}{d x}-\partial_{x} L_{\ell}^{(-\alpha-2)}(x)\right) \\
& \times\left((\alpha-\ell+1) L_{\ell}^{(-\alpha-2)}(x) L_{n}^{(\alpha+1)}(x)+x L_{\ell}^{(-\alpha-1)}(x) \partial_{x} L_{n}^{(\alpha+1)}(x)\right) \\
& \quad+L_{\ell}^{(-\alpha-1)}(x)\left((\alpha-\ell+2) L_{\ell}^{(-\alpha-3)}(x) L_{n-1}^{(\alpha+2)}(x)+x L_{\ell}^{(-\alpha-2)}(x) \partial_{x} L_{n-1}^{(\alpha+2)}(x)\right), \\
(\mathrm{B}): \quad 0= & \left(L_{\ell}^{(-\alpha-1)}(x)\left(x \frac{d}{d x}+\alpha+1-x\right)-x \partial_{x} L_{\ell}^{(-\alpha-1)}(x)\right) \\
& \times\left((\alpha-\ell+2) L_{\ell}^{(-\alpha-3)}(x) L_{n-1}^{(\alpha+2)}(x)+x L_{\ell}^{(-\alpha-2)}(x) \partial_{x} L_{n-1}^{(\alpha+2)}(x)\right) \\
& -n L_{\ell}^{(-\alpha-2)}(x)\left((\alpha-\ell+1) L_{\ell}^{(-\alpha-2)}(x) L_{n}^{(\alpha+1)}(x)+x L_{\ell}^{(-\alpha-1)}(x) \partial_{x} L_{n}^{(\alpha+1)}(x)\right),
\end{aligned}
$$

(J2): $\left(\alpha=g+\ell-\frac{1}{2}, \beta=h+\ell-\frac{1}{2}\right)$

$$
\begin{aligned}
(\mathrm{F}): \quad 0= & \left(P_{\ell}^{(-\alpha-2, \beta)}(x) \frac{d}{d x}-\partial_{x} P_{\ell}^{(-\alpha-2, \beta)}(x)\right)\left((\alpha-\ell+1) P_{\ell}^{(-\alpha-2, \beta)}(x) P_{n}^{(\alpha+1, \beta-1)}(x)\right. \\
& \left.-(1-x) P_{\ell}^{(-\alpha-1, \beta-1)}(x) \partial_{x} P_{n}^{(\alpha+1, \beta-1)}(x)\right)-\frac{1}{2}(n+\alpha+\beta+1) P_{\ell}^{(-\alpha-1, \beta-1)}(x) \\
& \times\left((\alpha-\ell+2) P_{\ell}^{(-\alpha-3, \beta+1)}(x) P_{n-1}^{(\alpha+2, \beta)}(x)\right. \\
& \left.-(1-x) P_{\ell}^{(-\alpha-2, \beta)}(x) \partial_{x} P_{n-1}^{(\alpha+2, \beta)}(x)\right) \\
(\mathrm{B}): \quad 0= & \left(P_{\ell}^{(-\alpha-1, \beta-1)}(x)\left(\left(1-x^{2}\right) \frac{d}{d x}+\beta-\alpha-(\alpha+\beta+2) x\right)\right.
\end{aligned}
$$




$$
\begin{aligned}
& \left.-\left(1-x^{2}\right) \partial_{x} P_{\ell}^{(-\alpha-1, \beta-1)}(x)\right)\left((\alpha-\ell+2) P_{\ell}^{(-\alpha-3, \beta+1)}(x) P_{n-1}^{(\alpha+2, \beta)}(x)\right. \\
& \left.-(1-x) P_{\ell}^{(-\alpha-2, \beta)}(x) \partial_{x} P_{n-1}^{(\alpha+2, \beta)}(x)\right)+2 n P_{\ell}^{(-\alpha-2, \beta)}(x) \\
& \times\left((\alpha-\ell+1) P_{\ell}^{(-\alpha-2, \beta)}(x) P_{n}^{(\alpha+1, \beta-1)}(x)\right. \\
& \left.-(1-x) P_{\ell}^{(-\alpha-1, \beta-1)}(x) \partial_{x} P_{n}^{(\alpha+1, \beta-1)}(x)\right) .
\end{aligned}
$$

We do not show those for the J1 polynomials, since J1 and J2 are related by the mirror image, $\eta \leftrightarrow-\eta$ and renaming of the coupling constants $g \leftrightarrow h$. Thus the corresponding identities are essentially the same. The derivative terms in the above identities (5.3)-(5.8) can be eliminated by using the forward shift relations (E.2) and (E.15) for the Laguerre and Jacobi polynomials.

In Appendix B, the forward and backward shift relations for the four types of $X_{\ell}$ polynomials are proven elementarily.

\section{Invariant polynomial subspace}

Another characterisation of polynomials satisfying differential equations is the existence of invariant polynomial subspaces [8]. For the Laguerre and Jacobi differential equations with $\widetilde{\mathcal{H}}_{0}(\boldsymbol{\lambda})$ (3.7), (3.8), the space $\mathcal{V}_{0, n}$ of degree $n$ polynomials in $\eta$ is invariant

$$
\begin{aligned}
& \widetilde{\mathcal{H}}_{0}(\boldsymbol{\lambda}) \mathcal{V}_{0, n} \subseteq \mathcal{V}_{0, n}, \quad \mathcal{V}_{0, n} \stackrel{\text { def }}{=} \operatorname{Span}\left[1, \eta, \ldots, \eta^{n}\right], \\
& \widetilde{\mathcal{H}}_{0}(\boldsymbol{\lambda}) \eta^{n}=\mathcal{E}_{n}(\boldsymbol{\lambda}) \eta^{n}+\text { lower orders. }
\end{aligned}
$$

However, $\mathcal{V}_{0, n}$ is obviously not invariant under $\widetilde{\mathcal{H}}_{\ell}(\boldsymbol{\lambda})(3.1),(3.9)-(3.12)$ :

$$
\widetilde{\mathcal{H}}_{\ell}(\boldsymbol{\lambda}) \mathcal{V}_{0, n} \nsubseteq \mathcal{V}_{0, n}, \quad \ell=1,2, \ldots
$$

Instead we have

$$
\begin{aligned}
& \widetilde{\mathcal{H}}_{\ell}(\boldsymbol{\lambda}) \mathcal{V}_{\ell, n} \subseteq \mathcal{V}_{\ell, n}, \quad \ell=1,2, \ldots, \\
& \mathcal{V}_{\ell, n} \stackrel{\text { def }}{=}\left\{\begin{array}{l}
\operatorname{Span}\left[\eta^{k} \xi_{\ell}(\eta ; g+1)-k \eta^{k-1} \xi_{\ell}(\eta ; g) ; k=0,1, \ldots, n\right], \\
\operatorname{Span}\left[\eta^{k}\left(\left(g+\frac{1}{2}\right) \xi_{\ell}(\eta ; g+1)+k \xi_{\ell}(\eta ; g)\right) ; k=0,1, \ldots, n\right],
\end{array}\right. \\
& \mathcal{V}_{\ell, n} \stackrel{\text { def }}{=}\left\{\begin{array}{r}
\operatorname{Span}\left[\eta^{k}\left(h+\frac{1}{2}\right) \xi_{\ell}(\eta ; g+1, h+1)\right. \\
\left.+k(1+\eta) \eta^{k-1} \xi_{\ell}(\eta ; g, h) ; k=0,1, \ldots, n\right], \\
\operatorname{Span}\left[\eta^{k}\left(g+\frac{1}{2}\right) \xi_{\ell}(\eta ; g+1, h+1)\right. \\
\left.-k(1-\eta) \eta^{k-1} \xi_{\ell}(\eta ; g, h) ; k=0,1, \ldots, n\right] .
\end{array}\right.
\end{aligned}
$$

As the basis vectors of the invariant polynomial subspace $\mathcal{V}_{\ell, n}$ for the $X_{\ell}$ Jacobi polynomials, one could have chosen for $k=0,1, \ldots, n$,

$$
\begin{aligned}
& (1-\eta)^{k-1}\left(\left(h+\frac{1}{2}\right)(1-\eta) \xi_{\ell}(\eta ; g+1, h+1)-k(1+\eta) \xi_{\ell}(\eta ; g, h)\right), \\
& (1+\eta)^{k}\left(\left(h+\frac{1}{2}\right) \xi_{\ell}(\eta ; g+1, h+1)+k \xi_{\ell}(\eta ; g, h)\right), \\
& (1-\eta)^{k}\left(\left(g+\frac{1}{2}\right) \xi_{\ell}(\eta ; g+1, h+1)+k \xi_{\ell}(\eta ; g, h)\right) .
\end{aligned}
$$

After overall rescaling, (6.5) and (6.7) go to those of the $X_{\ell}$ Laguerre polynomials (6.3) in the limit (E.28), as J1 $\rightarrow \mathrm{L} 1$ and $\mathrm{J} 2 \rightarrow \mathrm{L} 2$. As will be shown shortly these basis vectors (6.6) and (6.7) have simpler integration formulas than (6.4). It should be stressed that these basis vectors have the same common structure as the $X_{\ell}$ polynomials (2.8), (2.7),

$$
\Xi_{\ell, \boldsymbol{\lambda}}\left[p_{k}(\eta)\right]=d_{1}(\boldsymbol{\lambda}) \xi_{\ell}(\eta ; \boldsymbol{\lambda}+\boldsymbol{\delta}) p_{k}(\eta)-d_{2}(\eta) \xi_{\ell}(\eta ; \boldsymbol{\lambda}) \partial_{\eta} p_{k}(\eta) \in \mathcal{V}_{\ell, n}, \quad k=0,1, \ldots, n,(6.8)
$$


in which $p_{k}(\eta)$ is an arbitrary degree $k$ polynomial in $\eta$. These basis vectors are so chosen as not to develop any singularities at the zeros of $\xi_{\ell}(\eta ; \boldsymbol{\lambda})$ when applied by the forward shift operator $\mathcal{F}_{\ell}(\boldsymbol{\lambda})$ (3.3). In fact, for a polynomial $p(\eta), \mathcal{F}_{\ell}(\boldsymbol{\lambda})$ acts on $\Xi_{\ell, \boldsymbol{\lambda}}[p(\eta)]$ as

$$
\begin{aligned}
c_{\mathcal{F}}^{-1} \mathcal{F}_{\ell}(\boldsymbol{\lambda}) \Xi_{\ell, \boldsymbol{\lambda}}[p(\eta)]= & \left(d_{1}(\boldsymbol{\lambda}+\boldsymbol{\delta}) \partial_{\eta} p(\eta)-\partial_{\eta}\left(d_{2}(\eta) \partial_{\eta} p(\eta)\right)\right) \xi_{\ell}(\eta ; \boldsymbol{\lambda}+\boldsymbol{\delta}) \\
& +d_{2}(\eta) \partial_{\eta} p(\eta) \partial_{\eta} \xi_{\ell}(\eta ; \boldsymbol{\lambda}+\boldsymbol{\delta}),
\end{aligned}
$$

where we have used (2.20) to eliminate $\partial_{\eta} \xi_{\ell}(\eta ; \boldsymbol{\lambda})$. Since the backward shift operator $\mathcal{B}_{\ell}(\boldsymbol{\lambda})(3.4)$ does not cause any singularity at the zeros of $\xi_{\ell}(\eta ; \boldsymbol{\lambda})$ and the operator $\widetilde{\mathcal{H}}_{\ell}(\boldsymbol{\lambda})=\mathcal{B}_{\ell}(\boldsymbol{\lambda}) \mathcal{F}_{\ell}(\boldsymbol{\lambda})$ has the form (3.5), the application of $\widetilde{\mathcal{H}}_{\ell}(\boldsymbol{\lambda})$ on these basis vectors will result in polynomials in $\eta$.

Let us evaluate the action of the second order differential operator $\widetilde{\mathcal{H}}_{\ell}(\boldsymbol{\lambda})$ on $\Xi_{\ell, \boldsymbol{\lambda}}[p(\eta)]$ by applying the backward shift operator $\mathcal{B}_{\ell}(\boldsymbol{\lambda})$ on (6.9):

$$
\widetilde{\mathcal{H}}_{\ell}(\boldsymbol{\lambda}) \Xi_{\ell, \boldsymbol{\lambda}}[p(\eta)]=\xi_{\ell}(\eta ; \boldsymbol{\lambda}) X(\eta)+\xi_{\ell}(\eta ; \boldsymbol{\lambda}+\boldsymbol{\delta}) Y(\eta) .
$$

The coefficients $X(\eta)$ and $Y(\eta)$ can be written as

$$
\begin{aligned}
& X(\eta)=4 d_{2}(\eta) \partial_{\eta}\left(c_{2}(\eta) \partial_{\eta}^{2} p(\eta)+c_{1}\left(\eta, \boldsymbol{\lambda}^{\prime}\right) \partial_{\eta} p(\eta)\right)=-d_{2}(\eta) \partial_{\eta}\left(\widetilde{\mathcal{H}}_{0}\left(\boldsymbol{\lambda}^{\prime}\right) p(\eta)\right), \\
& Y(\eta)=-4 d_{1}(\boldsymbol{\lambda})\left(c_{2}(\eta) \partial_{\eta}^{2} p(\eta)+c_{1}\left(\eta, \boldsymbol{\lambda}^{\prime}\right) \partial_{\eta} p(\eta)\right)=d_{1}(\boldsymbol{\lambda}) \widetilde{\mathcal{H}}_{0}\left(\boldsymbol{\lambda}^{\prime}\right) p(\eta),
\end{aligned}
$$

where $\boldsymbol{\lambda}^{\prime}=\boldsymbol{\lambda}+\ell \boldsymbol{\delta}+\tilde{\boldsymbol{\delta}}$ and $\widetilde{\mathcal{H}}_{0}(\boldsymbol{\lambda})$ is defined in (3.6). Therefore the results can be expressed in quite a simple form as

$$
\widetilde{\mathcal{H}}_{\ell}(\boldsymbol{\lambda}) \Xi_{\ell, \boldsymbol{\lambda}}[p(\eta)]=\Xi_{\ell, \boldsymbol{\lambda}}\left[\widetilde{\mathcal{H}}_{0}(\boldsymbol{\lambda}+\ell \boldsymbol{\delta}+\tilde{\boldsymbol{\delta}}) p(\eta)\right] .
$$

By taking $p(\eta)=p_{k}(\eta)$, the above basis vectors satisfy

$$
\widetilde{\mathcal{H}}_{\ell}(\boldsymbol{\lambda}) \Xi_{\ell, \boldsymbol{\lambda}}\left[p_{k}(\eta)\right]=\Xi_{\ell, \boldsymbol{\lambda}}\left[\widetilde{\mathcal{H}}_{0}(\boldsymbol{\lambda}+\ell \boldsymbol{\delta}+\tilde{\boldsymbol{\delta}}) p_{k}(\eta)\right] \in \mathcal{V}_{\ell, n}, \quad k \leq n
$$

In other words, we have shown that $\mathcal{V}_{\ell, n}$ is an invariant polynomial subspace of the differential operator $\widetilde{\mathcal{H}}_{\ell}(\boldsymbol{\lambda})$ and we obtain, corresponding to (6.1),

$$
\widetilde{\mathcal{H}}_{\ell}(\boldsymbol{\lambda}) \Xi_{\ell, \boldsymbol{\lambda}}\left[p_{n}(\eta)\right]=\mathcal{E}_{n}(\boldsymbol{\lambda}+\ell \boldsymbol{\delta}) \Xi_{\ell, \boldsymbol{\lambda}}\left[p_{n}(\eta)\right]+\text { lower orders. }
$$

In particular, if we choose $p_{n}(\eta)$ as the eigenfunction $P_{n}(\eta ; \boldsymbol{\lambda}+\ell \boldsymbol{\delta}+\tilde{\boldsymbol{\delta}})$ of $\widetilde{\mathcal{H}}_{0}(\boldsymbol{\lambda}+\ell \boldsymbol{\delta}+\tilde{\boldsymbol{\delta}})$,

$$
\widetilde{\mathcal{H}}_{0}(\boldsymbol{\lambda}+\ell \boldsymbol{\delta}+\tilde{\boldsymbol{\delta}}) P_{n}(\eta ; \boldsymbol{\lambda}+\ell \boldsymbol{\delta}+\tilde{\boldsymbol{\delta}})=\mathcal{E}_{n}(\boldsymbol{\lambda}+\ell \boldsymbol{\delta}) P_{n}(\eta ; \boldsymbol{\lambda}+\ell \boldsymbol{\delta}+\tilde{\boldsymbol{\delta}}),
$$

then we find that $\Xi_{\ell, \boldsymbol{\lambda}}\left[P_{n}(\eta ; \boldsymbol{\lambda}+\ell \boldsymbol{\delta}+\tilde{\boldsymbol{\delta}})\right]$ is the eigenfunction of $\widetilde{\mathcal{H}}_{\ell}(\boldsymbol{\lambda})$,

$$
\widetilde{\mathcal{H}}_{\ell}(\boldsymbol{\lambda}) \Xi_{\ell, \boldsymbol{\lambda}}\left[P_{n}(\eta ; \boldsymbol{\lambda}+\ell \boldsymbol{\delta}+\tilde{\boldsymbol{\delta}})\right]=\mathcal{E}_{n}(\boldsymbol{\lambda}+\ell \boldsymbol{\delta}) \Xi_{\ell, \boldsymbol{\lambda}}\left[P_{n}(\eta ; \boldsymbol{\lambda}+\ell \boldsymbol{\delta}+\tilde{\boldsymbol{\delta}})\right],
$$

as given in (2.8). Note that the effect of $\tilde{\boldsymbol{\delta}}$ in the eigenvalue cancels out. This is another analytical proof for the explicit forms of the $X_{\ell}$ polynomials $P_{\ell, n}(\eta ; \boldsymbol{\lambda})$ as given $(2.8)$.

\section{$7 \quad$ Integration formulas}

Another well-known construction method of orthogonal polynomials is the Gram-Schmidt orthonormalisation of certain basis vectors under a given inner product specified by a weight function. Let us introduce two types of inner products $\langle *, *\rangle_{\ell, \lambda}$ and $(*, *)_{\lambda}$ :

$$
\langle p(\eta), q(\eta)\rangle_{\ell, \boldsymbol{\lambda}} \stackrel{\text { def }}{=} \int p(\eta) q(\eta) \mathcal{W}_{\ell}(\eta ; \boldsymbol{\lambda}) d \eta, \quad(p(\eta), q(\eta))_{\boldsymbol{\lambda}} \stackrel{\text { def }}{=} \int p(\eta) q(\eta) W(\eta ; \boldsymbol{\lambda}) d \eta,
$$


in which $p(\eta)$ and $q(\eta)$ are arbitrary functions and $\mathcal{W}_{\ell}(\eta ; \boldsymbol{\lambda})$ is the weight function for the $X_{\ell}$ polynomials, whereas $W(\eta ; \boldsymbol{\lambda})$ is the weight function for the Laguerre or Jacobi polynomials (2.11).

Here we present the integration formulas:

$$
\begin{aligned}
& \left\langle\Xi_{\ell, \boldsymbol{\lambda}}[p(\eta)], \Xi_{\ell, \boldsymbol{\lambda}}[q(\eta)]\right\rangle_{\ell, \boldsymbol{\lambda}} \\
& \quad=d_{1}(\boldsymbol{\lambda}) d_{3}(\boldsymbol{\lambda}+\ell \boldsymbol{\delta}, \ell)(p(\eta), q(\eta))_{\boldsymbol{\lambda}^{\prime}}+\frac{1}{4} c_{\mathcal{F}}^{2}\left(\partial_{\eta} p(\eta), \partial_{\eta} q(\eta)\right)_{\boldsymbol{\lambda}^{\prime}+\boldsymbol{\delta}}, \quad \text { or } \\
& \int \Xi_{\ell, \boldsymbol{\lambda}}[p(\eta)] \Xi_{\ell, \boldsymbol{\lambda}}[q(\eta)] \mathcal{W}_{\ell}(\eta ; \boldsymbol{\lambda}) d \eta \\
& \quad=\int\left(d_{1}(\boldsymbol{\lambda}) d_{3}(\boldsymbol{\lambda}+\ell \boldsymbol{\delta}, \ell) p(\eta) q(\eta) W\left(\eta ; \boldsymbol{\lambda}^{\prime}\right)+\frac{1}{4} c_{\mathcal{F}}^{2} \partial_{\eta} p(\eta) \partial_{\eta} q(\eta) W\left(\eta ; \boldsymbol{\lambda}^{\prime}+\boldsymbol{\delta}\right)\right) d \eta,
\end{aligned}
$$

where $\boldsymbol{\lambda}^{\prime}=\boldsymbol{\lambda}+\ell \boldsymbol{\delta}+\tilde{\boldsymbol{\delta}}$. For a proof, see Appendix C.

With this formula one can easily verify the orthogonality relation and the normalisation constants of the $X_{\ell}$ polynomials $(2.10),(2.13)$, (2.15) given in Section 2. One simply takes $p(\eta)=P_{n}\left(\eta ; \boldsymbol{\lambda}^{\prime}\right)$ and $q(\eta)=P_{m}\left(\eta ; \boldsymbol{\lambda}^{\prime}\right)$ for L1-J2. Then the two terms in (7.2) read

$$
\begin{aligned}
& \left(P_{n}\left(\eta ; \boldsymbol{\lambda}^{\prime}\right), P_{m}\left(\eta ; \boldsymbol{\lambda}^{\prime}\right)\right)_{\boldsymbol{\lambda}^{\prime}}=\int P_{n}\left(\eta ; \boldsymbol{\lambda}^{\prime}\right) P_{m}\left(\eta ; \boldsymbol{\lambda}^{\prime}\right) W\left(\eta ; \boldsymbol{\lambda}^{\prime}\right) d \eta=h_{n}\left(\boldsymbol{\lambda}^{\prime}\right) \delta_{n m}, \\
& c_{\mathcal{F}}^{2} \int \partial_{\eta} P_{n}\left(\eta ; \boldsymbol{\lambda}^{\prime}\right) \partial_{\eta} P_{m}\left(\eta ; \boldsymbol{\lambda}^{\prime}\right) W\left(\eta ; \boldsymbol{\lambda}^{\prime}+\boldsymbol{\delta}\right) d \eta=f_{n}\left(\boldsymbol{\lambda}^{\prime}\right)^{2} h_{n-1}\left(\boldsymbol{\lambda}^{\prime}+\boldsymbol{\delta}\right) \delta_{n m}
\end{aligned}
$$

where we have used (2.23). Therefore, from (2.8), the normalisation constant $h_{\ell, n}$ is expressed as

$$
d_{0}(n, \boldsymbol{\lambda})^{2} h_{\ell, n}(\boldsymbol{\lambda})=d_{1}(\boldsymbol{\lambda}) d_{3}(\boldsymbol{\lambda}+\ell \boldsymbol{\delta}, \ell) h_{n}\left(\boldsymbol{\lambda}^{\prime}\right)+\frac{1}{4} f_{n}\left(\boldsymbol{\lambda}^{\prime}\right)^{2} h_{n-1}\left(\boldsymbol{\lambda}^{\prime}+\boldsymbol{\delta}\right)
$$

where $\boldsymbol{\lambda}^{\prime}=\boldsymbol{\lambda}+\ell \boldsymbol{\delta}+\tilde{\boldsymbol{\delta}}$ and $h_{-1}(\boldsymbol{\lambda}) \stackrel{\text { def }}{=} 0$. This formula with $h_{n}(2.12)$ and (2.14) gives (2.13) and (2.15).

The integration formula reveals another important property of the invariant subspaces, the orthogonality, $\mathcal{V}_{\ell, m} \perp P_{\ell, n}(\eta ; \boldsymbol{\lambda}), m<n$ :

$$
\left\langle\Xi_{\ell, \boldsymbol{\lambda}}\left[p_{m}(\eta)\right], P_{\ell, n}(\eta ; \boldsymbol{\lambda})\right\rangle_{\ell, \boldsymbol{\lambda}}=0, \quad m<n,
$$

which is a simple consequence of (2.8) and the well known fact

$$
\left(p_{m}(\eta), P_{n}(\eta ; \boldsymbol{\lambda})\right)_{\boldsymbol{\lambda}}=0, \quad m<n
$$

\section{Gram-Schmidt orthonormalisation}

As for the direct application of Gram-Schmidt orthonormalisation, one orthonormalises $\left\{\Xi_{\ell, \boldsymbol{\lambda}}\left[p_{n}(\eta)\right]\right\}$ in (6.8) with respect to the inner product $\langle *, *\rangle_{\ell, \boldsymbol{\lambda}}$. The following choice of the function $p_{n}$ is made:

$$
p_{n}(\eta)= \begin{cases}\eta^{n}, & (\mathrm{~L}) \\ (1 \pm \eta)^{n}, & (\mathrm{~J} 1 / \mathrm{J} 2)\end{cases}
$$

with which the inner products $\left(p_{n}(\eta), p_{m}(\eta)\right)_{\lambda^{\prime}}$ are easily expressed in terms of gamma functions. This corresponds to the choice of the basis vectors in (6.6) and (6.7). 
The Gram-Schmidt orthonormalisation procedure at the $n$-th step determines $P_{\ell, n}(\eta ; \boldsymbol{\lambda})$ by the following formula

$$
P_{\ell, n}(\eta ; \boldsymbol{\lambda}) \propto \Xi_{\ell, \boldsymbol{\lambda}}\left[p_{n}(\eta)\right]-\sum_{m=0}^{n-1} P_{\ell, m}(\eta ; \boldsymbol{\lambda})\left\langle P_{\ell, m}(\eta ; \boldsymbol{\lambda}), \Xi_{\ell, \boldsymbol{\lambda}}\left[p_{n}(\eta)\right]\right\rangle_{\ell, \boldsymbol{\lambda}} h_{\ell, m}(\boldsymbol{\lambda})^{-1},
$$

which is to be compared with the procedure for the undeformed $(\ell=0)$ polynomial

$$
P_{n}\left(\eta ; \boldsymbol{\lambda}^{\prime}\right) \propto p_{n}(\eta)-\sum_{m=0}^{n-1} P_{m}\left(\eta ; \boldsymbol{\lambda}^{\prime}\right)\left(P_{m}\left(\eta ; \boldsymbol{\lambda}^{\prime}\right), p_{n}(\eta)\right)_{\boldsymbol{\lambda}^{\prime}} h_{m}\left(\boldsymbol{\lambda}^{\prime}\right)^{-1},
$$

where $\boldsymbol{\lambda}^{\prime}=\boldsymbol{\lambda}+\ell \boldsymbol{\delta}+\tilde{\boldsymbol{\delta}}$. These two orthonormalisations are essentially the same. In fact, by applying $\Xi_{\ell, \boldsymbol{\lambda}}[\cdot]$ to $(8.2)$ and using $(2.8)$, we have

$$
P_{\ell, n}(\eta ; \boldsymbol{\lambda}) \propto \Xi_{\ell, \boldsymbol{\lambda}}\left[p_{n}(\eta)\right]-\sum_{m=0}^{n-1} P_{\ell, m}(\eta ; \boldsymbol{\lambda}) d_{0}(m, \boldsymbol{\lambda})\left(P_{m}\left(\eta ; \boldsymbol{\lambda}^{\prime}\right), p_{n}(\eta)\right)_{\boldsymbol{\lambda}^{\prime}} h_{m}\left(\boldsymbol{\lambda}^{\prime}\right)^{-1}
$$

Comparing these we obtain

$$
\begin{aligned}
& \left\langle P_{\ell, m}(\eta ; \boldsymbol{\lambda}), \Xi_{\ell, \boldsymbol{\lambda}}\left[p_{n}(\eta)\right]\right\rangle_{\ell, \boldsymbol{\lambda}} h_{\ell, m}(\boldsymbol{\lambda})^{-1} \\
& \quad=d_{0}(m, \boldsymbol{\lambda})\left(P_{m}\left(\eta ; \boldsymbol{\lambda}^{\prime}\right), p_{n}(\eta)\right)_{\boldsymbol{\lambda}^{\prime}} h_{m}\left(\boldsymbol{\lambda}^{\prime}\right)^{-1}, \quad m=0, \ldots, n-1 .
\end{aligned}
$$

In other words, the Gram-Schmidt orthonormalisation for the undeformed $(\ell=0)$ polynomials

$$
p_{n}(\eta) \rightarrow P_{n}\left(\eta ; \boldsymbol{\lambda}^{\prime}\right)
$$

provides that for the $X_{\ell}$ polynomials, too.

\section{$9 \quad$ Generating functions}

Generating functions for orthogonal polynomials have played another important role in classical analysis. Let us define generating functions for the $X_{\ell}$ polynomials $P_{\ell, n}$ and for the undeformed polynomials $P_{n}$,

$$
G_{\ell}(t, \eta ; \boldsymbol{\lambda}) \stackrel{\text { def }}{=} \sum_{n=0}^{\infty} t^{n} P_{\ell, n}(\eta ; \boldsymbol{\lambda}), \quad G(t, \eta ; \boldsymbol{\lambda}) \stackrel{\text { def }}{=} G_{0}(t, \eta ; \boldsymbol{\lambda})=\sum_{n=0}^{\infty} t^{n} P_{n}(\eta ; \boldsymbol{\lambda}) .
$$

The latter is quite well known

$$
G(t, \eta ; \boldsymbol{\lambda})=\left\{\begin{array}{l}
G^{\left(g-\frac{1}{2}\right)}(t, \eta) \\
G^{\left(g-\frac{1}{2}, h-\frac{1}{2}\right)}(t, \eta)
\end{array}\right.
$$

where $G^{(\alpha)}(t, x)$ and $G^{(\alpha, \beta)}(t, x)$ are given in (E.8) and (E.21). Since $P_{\ell, n}(\eta ; \boldsymbol{\lambda})$ is expressed linearly in terms of $P_{n}\left(\eta ; \boldsymbol{\lambda}^{\prime}\right)(2.5)\left(\boldsymbol{\lambda}^{\prime}=\boldsymbol{\lambda}+\ell \boldsymbol{\delta}+\tilde{\boldsymbol{\delta}}\right)$, the generating function $G_{\ell}(t, \eta ; \boldsymbol{\lambda})$ is expressed simply in terms of the known $G\left(t, \eta ; \boldsymbol{\lambda}^{\prime}\right)$ :

$$
\begin{aligned}
d_{0}\left(t \partial_{t}, \boldsymbol{\lambda}\right) G_{\ell}(t, \eta ; \boldsymbol{\lambda}) & =\sum_{n=0}^{\infty} t^{n} d_{0}(n, \boldsymbol{\lambda}) P_{\ell, n}(\eta ; \boldsymbol{\lambda}) \\
& =d_{1}(\boldsymbol{\lambda}) \xi_{\ell}(\eta ; \boldsymbol{\lambda}+\boldsymbol{\delta}) G\left(t, \eta ; \boldsymbol{\lambda}^{\prime}\right)-d_{2}(\eta) \xi_{\ell}(\eta ; \boldsymbol{\lambda}) \partial_{\eta} G\left(t, \eta ; \boldsymbol{\lambda}^{\prime}\right) .
\end{aligned}
$$


The forward shift relation (2.23) implies

$$
\partial_{\eta} G(t, \eta ; \boldsymbol{\lambda})=c_{\mathcal{F}}^{-1} t f_{t \partial_{t}+1}(\boldsymbol{\lambda}) G(t, \eta ; \boldsymbol{\lambda}+\boldsymbol{\delta}) .
$$

Here we present the concrete forms of the generating functions:

$$
\begin{aligned}
d_{0}\left(t \partial_{t}, \boldsymbol{\lambda}\right) & G_{\ell}(t, \eta ; \boldsymbol{\lambda}) \\
= & \left\{\begin{array}{l}
\left(L_{\ell}^{\left(g+\ell-\frac{1}{2}\right)}(-\eta)+\frac{t}{1-t} L_{\ell}^{\left(g+\ell-\frac{3}{2}\right)}(-\eta)\right) G^{\left(g+\ell-\frac{3}{2}\right)}(t, \eta), \\
\left(\left(g+\frac{1}{2}\right) L_{\ell}^{\left(-g-\ell-\frac{3}{2}\right)}(\eta)-\frac{t \eta}{1-t} L_{\ell}^{\left(-g-\ell-\frac{1}{2}\right)}(\eta)\right) G^{\left(g+\ell+\frac{1}{2}\right)}(t, \eta), \\
\left(\left(h+\frac{1}{2}\right) P_{\ell}^{\left(g+\ell-\frac{1}{2},-h-\ell-\frac{3}{2}\right)}(\eta)+\frac{(1+\eta) t}{R}\left(\frac{1}{R}+\frac{g+\ell-\frac{3}{2}}{1+R-t}+\frac{h+\ell+\frac{1}{2}}{1+R+t}\right)\right. \\
\left.\quad \times P_{\ell}^{\left(g+\ell-\frac{3}{2},-h-\ell-\frac{1}{2}\right)}(\eta)\right) G^{\left(g+\ell-\frac{3}{2}, h+\ell+\frac{1}{2}\right)}(t, \eta), \\
\left(\left(g+\frac{1}{2}\right) P_{\ell}^{\left(-g-\ell-\frac{3}{2}, h+\ell-\frac{1}{2}\right)}(\eta)-\frac{(1-\eta) t}{R}\left(\frac{1}{R}+\frac{g+\ell+\frac{1}{2}}{1+R-t}+\frac{h+\ell-\frac{3}{2}}{1+R+t}\right)\right. \\
\left.\quad \times P_{\ell}^{\left(-g-\ell-\frac{1}{2}, h+\ell-\frac{3}{2}\right)}(\eta)\right) G^{\left(g+\ell+\frac{1}{2}, h+\ell-\frac{3}{2}\right)}(t, \eta),
\end{array}\right.
\end{aligned}
$$

where $R \stackrel{\text { def }}{=} \sqrt{1-2 \eta t+t^{2}}$.

Next let us introduce the double generating function, that is the generating function of the generating functions $G_{\ell}(t, \eta ; \boldsymbol{\lambda})$ :

$$
\mathcal{G}(s, t, \eta ; \boldsymbol{\lambda}) \stackrel{\text { def }}{=} \sum_{\ell=0}^{\infty} s^{\ell} G_{\ell}(t, \eta ; \boldsymbol{\lambda}) .
$$

For L1 and L2 cases, the explicit forms are:

$$
\begin{aligned}
&(\mathrm{L} 1): \quad \mathcal{G}(s, t, \eta ; g)= 2^{g-\frac{3}{2}}((2-t) \sqrt{1-t}+t \sqrt{1-t-4 s}) \\
& \times \frac{e^{-\frac{t}{1-t} \eta+\frac{1}{4} s^{-1}}(\sqrt{1-t}-\sqrt{1-t-4 s})^{2} \eta}{\sqrt{1-t-4 s} \sqrt{1-t}^{g+\frac{3}{2}}(\sqrt{1-t}+\sqrt{1-t-4 s})^{g-\frac{1}{2}}}, \\
&(\mathrm{~L} 2): \quad d_{0}\left(t \partial_{t}, g\right) \mathcal{G}(s, t, \eta ; g)=\left(g+\frac{1}{2}-\frac{t(1-t+s)}{(1-t)^{2}} \eta\right) \frac{e^{-\frac{s+t}{1-t} \eta}}{(1-t+s)^{g+\frac{3}{2}}}
\end{aligned}
$$

which are obtained by using the two shifted generating functions (E.9) and (E.10) in Appendix E.1. It is a good challenge to derive the double generating functions for the $X_{\ell}$ Jacobi polynomials.

\section{Three term recurrence relations}

Three term recurrence relations are one of the most fundamental characteristics of the ordinary orthogonal polynomials of one variable. Obviously the exceptional orthogonal polynomials do not satisfy these relations. Nevertheless, being deformations of ordinary orthogonal polynomials, the $X_{\ell}$ polynomials are expected to retain certain reminiscent properties of the three term recurrence.

Here we present a simple modification of the three term recurrence relations valid for the $X_{\ell}$ polynomials. Its relevance is, however, as yet unclear. Let us denote the three term recurrence relation for the Laguerre or the Jacobi polynomials as

$$
\eta P_{n}(\eta ; \boldsymbol{\lambda})=A_{n}(\boldsymbol{\lambda}) P_{n+1}(\eta ; \boldsymbol{\lambda})+B_{n}(\boldsymbol{\lambda}) P_{n}(\eta ; \boldsymbol{\lambda})+C_{n}(\boldsymbol{\lambda}) P_{n-1}(\eta ; \boldsymbol{\lambda}),
$$


in which the explicit forms of the coefficients $A_{n}, B_{n}$ and $C_{n}$ can be read from (E.5) and (E.18) in Appendix E.1 and Appendix E.2. As a substitute of the above three term recurrence relations, we expect that a certain element in the degree $\ell+n+1$ invariant polynomial subspace $\mathcal{V}_{\ell, n+1}$ (6.3), (6.4), which is related to $\eta P_{\ell, n}\left(\eta ; \boldsymbol{\lambda}^{\prime}\right)$, to be expressed in terms of $P_{\ell, n+1}(\eta ; \boldsymbol{\lambda}), P_{\ell, n}(\eta ; \boldsymbol{\lambda})$ and $P_{\ell, n-1}(\eta ; \boldsymbol{\lambda})$. From $(2.8)$ this can be achieved as

$$
\begin{aligned}
\Xi_{\ell, \boldsymbol{\lambda}}\left[\eta P_{n}\left(\eta ; \boldsymbol{\lambda}^{\prime}\right)\right]= & A_{n}\left(\boldsymbol{\lambda}^{\prime}\right) d_{0}(n+1, \boldsymbol{\lambda}) P_{\ell, n+1}(\eta ; \boldsymbol{\lambda})+B_{n}\left(\boldsymbol{\lambda}^{\prime}\right) d_{0}(n, \boldsymbol{\lambda}) P_{\ell, n}(\eta ; \boldsymbol{\lambda}) \\
& +C_{n}\left(\boldsymbol{\lambda}^{\prime}\right) d_{0}(n-1, \boldsymbol{\lambda}) P_{\ell, n-1}(\eta ; \boldsymbol{\lambda}),
\end{aligned}
$$

where $\boldsymbol{\lambda}^{\prime}=\boldsymbol{\lambda}+\ell \boldsymbol{\delta}+\tilde{\boldsymbol{\delta}}$

\section{Zeros of $X_{\ell}$ polynomials}

The zeros of orthogonal polynomials have always attracted the interest of researchers. In the case of $X_{\ell}$ polynomials $P_{\ell, n}(\eta ; \boldsymbol{\lambda})$, it has $n$ zeros in the domain where the weight function is defined, that is $(0, \infty)$ for the L1 and L2 polynomials and $(-1,1)$ for the J1 and J2 polynomials. See for example, Section 5.4 of [19]. The behaviour of these zeros are the same as those of other ordinary orthogonal polynomials. This is guaranteed by the oscillation theorem of the one-dimensional quantum mechanics, since $P_{\ell, n}(\eta ; \boldsymbol{\lambda})$ are obtained as the polynomial part of the eigenfunctions of a shape invariant quantum mechanical problem $[1,2]$.

Here we discuss the location of the extra $\ell$ zeros of the exceptional orthogonal polynomials, which lie in various different positions for the different types of polynomials. So far we have verified by direct calculation for lower $\ell$ and $n$ : The $\ell$ extra zeros of L1 polynomials are on the negative real line $(-\infty, 0)$. Those of the $\mathrm{L} 2 X_{\ell \text { :odd }}$ polynomials are 1 real negative zero which lies to the left of the remaining $\frac{1}{2}(\ell-1)$ pairs of complex conjugate roots. The L $2 X_{\ell \text { :even }}$ polynomials have $\frac{1}{2} \ell$ pairs of complex conjugate roots. For L2, these $\ell$ additional roots lie to the left of the $n$ real zeros.

The situations for the $X_{\ell}$ Jacobi polynomials are a bit more complicated. The J1 $X_{\ell \text { :odd }}$ polynomials have 1 real negative root which lies to the left of the remaining $\frac{1}{2}(\ell-1)$ pairs of complex conjugate roots with negative real parts. The J1 $X_{\ell \text { :even }}$ polynomials have $\frac{1}{2} \ell$ pairs of complex conjugate roots with negative real parts. For J1, some of the complex roots have real parts between -1 and 0 . The $\mathrm{J} 2 X_{\ell \text { :odd }}$ polynomials have 1 real positive root which lies to the right of the remaining $\frac{1}{2}(\ell-1)$ pairs of complex conjugate roots with positive real parts. The J2

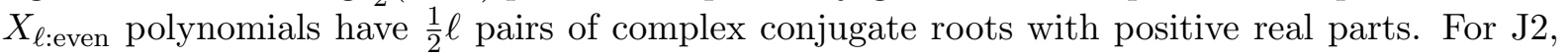
some of the complex roots have real parts between 0 and 1.

\section{Summary and comments}

We have given an in-depth study of the properties of the exceptional $\left(X_{\ell}\right)$ polynomials discovered recently in $[1,2,15]$. Our main focus is the derivation of certain equivalent but much simpler looking forms of the $X_{\ell}$ polynomials. The derivation is based on the analysis of the second order differential equations for the $X_{\ell}$ polynomials within the framework of the Fuchsian differential equations in the entire complex plane. These new forms of the $X_{\ell}$ polynomials allow easy verification of the actions of the forward and backward shift operators on the $X_{\ell}$ polynomials, and provide direct derivation of the Rodrigues formulas and the generating functions. The structure of the invariant polynomial subspaces under the Fuchsian differential operators is elucidated. The bases of the invariant polynomial subspaces provide a simple substitute of the three term recurrence relations. The Gram-Schmidt construction of the $X_{\ell}$ polynomials starting from the above bases is demonstrated with the help of an integration formula. The properties of the extra zeros of the $X_{\ell}$ polynomials are discussed. Some technical details are 
relegated to the Appendices. The proof of the equivalence of the new and original forms of the $X_{\ell}$ polynomials is given. Simple proofs of the forward and backward shift operations are shown. The integration formula is elementarily proven. Various fundamental formulas of the Laguerre and Jacobi polynomials are supplied for easy reference.

Let us mention that the same method, deformation in terms of a degree $\ell$ eigenpolynomial, applied to the discrete quantum mechanical Hamiltonians for the Wilson and Askey-Wilson polynomials produced two sets of infinitely many shape invariant systems together with exceptional $\left(X_{\ell}\right)$ Wilson and Askey-Wilson polynomials $(\ell=1,2, \ldots)$ [16]. It will be interesting to carry out the same analysis in these discrete cases.

Finally, concerning the issue of global solutions of Fuchsian differential equations, we would like to make a comment on the well-known theorem by Heine-Stieltjes [20]. It asserts the existence of a polynomial solution for the differential equation

$$
A(x) \frac{d^{2}}{d x^{2}} y(x)+B(x) \frac{d}{d x} y(x)+C(x) y(x)=0,
$$

in which only two coefficient functions $A(x)$ and $B(x)$ are specified. They are degree $p+1$ and $p$ polynomials, respectively. In this case a polynomial $C(x)$ of degree $p-1$ is not given at the beginning but is determined so that the equation admits a degree $n$ polynomial solution. Thus the problem setting is not a proper Fuchsian differential equation and the process of determining $C(x)$ and the polynomial solution is purely algebraic. We consider these polynomial solutions do not qualify to be 'global solutions' of the ordinary Fuchsian equations.

After this paper was arXived, some of the results were re-derived in terms of the DarbouxCrum transformations [17, 18].

\section{A Equivalence of different forms of $X_{\ell}$ polynomials}

The exceptional Jacobi polynomial for the trigonometric DPT presented in [1], namely J2 exceptional polynomial, is

$$
\begin{aligned}
P_{\ell, n}(\eta ; \boldsymbol{\lambda}) \stackrel{\text { def }}{=} & \left(\xi_{\ell}(\eta ; g+1, h+1)+\frac{2 n(-g+h+\ell-1) \xi_{\ell-1}(\eta ; g, h+2)}{(-g+h+2 \ell-2)(g+h+2 n+2 \ell-1)}\right. \\
& \left.-\frac{n(2 h+4 \ell-3) \xi_{\ell-2}(\eta ; g+1, h+3)}{(2 g+2 n+1)(-g+h+2 \ell-2)}\right) P_{n}(\eta ; \boldsymbol{\lambda}+\ell \boldsymbol{\delta}) \\
& +\frac{(-g+h+\ell-1)(2 g+2 n+2 \ell-1)}{(2 g+2 n+1)(g+h+2 n+2 \ell-1)} \xi_{\ell-1}(\eta ; g, h+2) P_{n-1}(\eta ; \boldsymbol{\lambda}+\ell \boldsymbol{\delta}) .
\end{aligned}
$$

In this paper we have presented it in a much simpler form in (2.3) (J2)

$$
\begin{aligned}
P_{\ell, n}(\eta ; \boldsymbol{\lambda})=\frac{1}{n+g+\frac{1}{2}}( & \left(g+\frac{1}{2}\right) \xi_{\ell}(\eta ; g+1, h+1) P_{n}(\eta ; g+\ell+1, h+\ell-1) \\
& \left.-(1-\eta) \xi_{\ell}(\eta ; g, h) \partial_{\eta} P_{n}(\eta ; g+\ell+1, h+\ell-1)\right) .
\end{aligned}
$$

In the following we write them as $P_{\ell, n}^{\text {org }}(\eta ; \boldsymbol{\lambda})$ and $P_{\ell, n}^{\text {new }}(\eta ; \boldsymbol{\lambda})$, respectively and show that they are in fact equal by using various identities of the Jacobi polynomials. For lower $\ell$ and $n$ the equality can be verified by direct calculation.

We fix $\ell$ and use new parameters $\alpha$ and $\beta$ instead of $g$ and $h$,

$$
\alpha \stackrel{\text { def }}{=} g+\ell-\frac{1}{2}, \quad \beta \stackrel{\text { def }}{=} h+\ell-\frac{1}{2} .
$$


By using the forward shift relation for the Jacobi polynomial (E.15), the polynomials $\xi_{\ell}$ and $P_{\ell, n}$ are expressed as

$$
\begin{aligned}
& \xi_{\ell}(\eta ; \boldsymbol{\lambda})=P_{\ell}^{(-\alpha-1, \beta-1)}(\eta), \quad \xi_{\ell-1}(\eta ; \boldsymbol{\lambda})=P_{\ell-1}^{(-\alpha, \beta-2)}(\eta), \\
& \xi_{\ell-2}(\eta ; \boldsymbol{\lambda})=P_{\ell-2}^{(-\alpha+1, \beta-3)}(\eta), \\
& P_{\ell, n}^{\mathrm{org}}(\eta ; \boldsymbol{\lambda})=\left(P_{\ell}^{(-\alpha-2, \beta)}(\eta)+\frac{2 n(\ell-\alpha+\beta-1) P_{\ell-1}^{(-\alpha, \beta)}(\eta)}{(2 \ell-\alpha+\beta-2)(2 n+\alpha+\beta)}\right. \\
& \left.\quad-\frac{n(\beta+\ell-1) P_{\ell-2}^{(-\alpha, \beta)}(\eta)}{(\alpha+n-\ell+1)(2 \ell-\alpha+\beta-2)}\right) P_{n}^{(\alpha, \beta)}(\eta) \\
& \quad+\frac{(\ell-\alpha+\beta-1)(\alpha+n)}{(\alpha+n-\ell+1)(2 n+\alpha+\beta)} P_{\ell-1}^{(-\alpha, \beta)}(\eta) P_{n-1}^{(\alpha, \beta)}(\eta), \\
& P_{\ell, n}^{\text {new }}(\eta ; \boldsymbol{\lambda})=\frac{1}{\alpha+n-\ell+1}\left((\alpha-\ell+1) P_{\ell}^{(-\alpha-2, \beta)}(\eta) P_{n}^{(\alpha+1, \beta-1)}(\eta)\right. \\
& \left.\quad-\frac{1}{2}(n+\alpha+\beta+1)(1-\eta) P_{\ell}^{(-\alpha-1, \beta-1)}(\eta) P_{n-1}^{(\alpha+2, \beta)}(\eta)\right) .
\end{aligned}
$$

Here we provide the proof for the equivalence of the two expressions (A.1) and (A.2) step by step:

$$
\begin{aligned}
& P_{\ell, n}^{\mathrm{org}}(\eta ; \boldsymbol{\lambda}) \stackrel{(\mathrm{i})}{=}\left(\frac{(\ell+\beta)(1-\eta) P_{\ell-1}^{(-\alpha, \beta)}(\eta)+(\alpha+1)(1+\eta) P_{\ell-1}^{(-\alpha-1, \beta+1)}(\eta)}{-2 \ell}\right. \\
& +\frac{2 n(\ell-\alpha+\beta-1) P_{\ell-1}^{(-\alpha, \beta)}(\eta)}{(2 \ell-\alpha+\beta-2)(2 n+\alpha+\beta)}-\frac{n(\beta+\ell-1)}{(\alpha+n-\ell+1)(2 \ell-\alpha+\beta-2)} \\
& \left.\times \frac{2(\ell-1) P_{\ell-1}^{(-\alpha, \beta)}(\eta)-(-\alpha+\beta+2 \ell-2)(1+\eta)\left(P_{\ell-1}^{(-\alpha, \beta)}(\eta)-P_{\ell-1}^{(-\alpha-1, \beta+1)}(\eta)\right)}{-2(\beta+\ell-1)}\right) \\
& \times P_{n}^{(\alpha, \beta)}(\eta)+\frac{(\ell-\alpha+\beta-1)(\alpha+n)}{(\alpha+n-\ell+1)(2 n+\alpha+\beta)} P_{\ell-1}^{(-\alpha, \beta)}(\eta) \\
& \times \frac{2 n P_{n}^{(\alpha, \beta)}(\eta)-(\alpha+\beta+2 n)(1-\eta)\left(P_{n}^{(\alpha, \beta)}(\eta)-P_{n}^{(\alpha+1, \beta-1)}(\eta)\right)}{2(\alpha+n)} \\
& =\frac{1}{\alpha+n-\ell+1}\left(\frac{1}{2}(\ell-\alpha+\beta-1)(1-\eta) P_{\ell-1}^{(-\alpha, \beta)}(\eta) P_{n}^{(\alpha+1, \beta-1)}(\eta)\right. \\
& \left.+\frac{n+\alpha+1}{2 \ell}\left(-(\alpha-\ell+1)(1+\eta) P_{\ell-1}^{(-\alpha-1, \beta+1)}(\eta)-\beta(1-\eta) P_{\ell-1}^{(-\alpha, \beta)}(\eta)\right) P_{n}^{(\alpha, \beta)}(\eta)\right) \\
& =\frac{1}{\alpha+n-\ell+1}\left((\alpha-\ell+1) \frac{(\ell+\beta)(1-\eta) P_{\ell-1}^{(-\alpha, \beta)}(\eta)+(\alpha+1)(1+\eta) P_{\ell-1}^{(-\alpha-1, \beta+1)}(\eta)}{-2 \ell}\right. \\
& \times P_{n}^{(\alpha+1, \beta-1)}(\eta)+\frac{(\ell-\alpha-1)(1+\eta) P_{\ell-1}^{(-\alpha-1, \beta+1)}(\eta)-\beta(1-\eta) P_{\ell-1}^{(-\alpha, \beta)}(\eta)}{2 \ell} \\
& \left.\times\left((n+\alpha+1) P_{n}^{(\alpha, \beta)}(\eta)-(\alpha+1) P_{n}^{(\alpha+1, \beta-1)}(\eta)\right)\right) \\
& \stackrel{\text { (ii) }}{=} \frac{1}{\alpha+n-\ell+1}\left((\alpha-\ell+1) P_{\ell}^{(-\alpha-2, \beta)}(\eta) P_{n}^{(\alpha+1, \beta-1)}(\eta)\right. \\
& \left.+P_{\ell}^{(-\alpha-1, \beta-1)}(\eta)\left((n+\alpha+1) P_{n}^{(\alpha, \beta)}(\eta)-(\alpha+1) P_{n}^{(\alpha+1, \beta-1)}(\eta)\right)\right)
\end{aligned}
$$




$$
\begin{aligned}
& \stackrel{\text { (iii) }}{=} \frac{1}{\alpha+n-\ell+1}\left((\alpha-\ell+1) P_{\ell}^{(-\alpha-2, \beta)}(\eta) P_{n}^{(\alpha+1, \beta-1)}(\eta)\right. \\
& \left.\quad+P_{\ell}^{(-\alpha-1, \beta-1)}(\eta) \frac{-1}{2}(n+\alpha+\beta+1)(1-\eta) P_{n-1}^{(\alpha+2, \beta)}(\eta)\right)=P_{\ell, n}^{\text {new }}(\eta ; \boldsymbol{\lambda}),
\end{aligned}
$$

where we have used (E.23), (E.27) and (E.24) in (i), (E.23) and (E.26) in (ii), and (E.25) in (iii).

The other exceptional polynomials, the J1 case is obtained from the above J2 case by (E.14). The equality of the exceptional Laguerre polynomials given in $[1,2]$ and those given in this paper (2.1) will not be given here, since the L1 and L2 cases are obtained from the J1 and J2 cases by the limit (E.28).

\section{B Forward and backward shift relations}

Here we provide proofs for the forward (4.3) and backward (4.4) shift relations which apply equally for the four types of $X_{\ell}$ polynomials. The method is elementary based on various identities of the Laguerre and Jacobi polynomials.

The forward shift relation (4.3) is equivalent to a polynomial identity (5.1). By using $d_{0}(n-$ $1, \boldsymbol{\lambda}+\boldsymbol{\delta})=d_{0}(n, \boldsymbol{\lambda}), f_{n}(\boldsymbol{\lambda}+\tilde{\boldsymbol{\delta}})=f_{n}(\boldsymbol{\lambda})$ and (2.23), it is easy to show that r.h.s. of (5.1) can be factorised $\partial_{\eta} P_{n}(\eta ; \boldsymbol{\lambda}+\ell \boldsymbol{\delta}+\tilde{\boldsymbol{\delta}}) \times(\cdots)$. The forward shift relation is thus equivalent to $(\cdots)=0$, namely,

$$
\begin{aligned}
0= & d_{1}(\boldsymbol{\lambda}) \xi_{\ell}(\eta ; \boldsymbol{\lambda}+\boldsymbol{\delta})^{2}-d_{1}(\boldsymbol{\lambda}+\boldsymbol{\delta}) \xi_{\ell}(\eta ; \boldsymbol{\lambda}) \xi_{\ell}(\eta ; \boldsymbol{\lambda}+2 \boldsymbol{\delta})-\partial_{\eta} d_{2}(\eta) \xi_{\ell}(\eta ; \boldsymbol{\lambda}) \xi_{\ell}(\eta ; \boldsymbol{\lambda}+\boldsymbol{\delta}) \\
& +d_{2}(\eta) \xi_{\ell}(\eta ; \boldsymbol{\lambda}) \partial_{\eta} \xi_{\ell}(\eta ; \boldsymbol{\lambda}+\boldsymbol{\delta})-d_{2}(\eta) \partial_{\eta} \xi_{\ell}(\eta ; \boldsymbol{\lambda}) \xi_{\ell}(\eta ; \boldsymbol{\lambda}+\boldsymbol{\delta}) .
\end{aligned}
$$

This is a polynomial identity of degree $2 \ell$ and it is quadratic in the Laguerre/Jacobi polynomials. This identity can be proven elementarily by using $d_{1}(\boldsymbol{\lambda})-d_{1}(\boldsymbol{\lambda}+\boldsymbol{\delta})=\partial_{\eta} d_{2}(\eta)$ and (2.20) to eliminate $\xi_{\ell}(\eta ; \boldsymbol{\lambda}+2 \boldsymbol{\delta})$ and $\partial_{\eta} \xi_{\ell}(\eta ; \boldsymbol{\lambda})$.

The backward shift relation (4.4) is equivalent to a polynomial identity (5.2). By using $d_{0}(n-1, \boldsymbol{\lambda}+\boldsymbol{\delta})=d_{0}(n, \boldsymbol{\lambda})$ and $b_{n-1}(\boldsymbol{\lambda})=-2 n$, the r.h.s. of (5.2) becomes

$$
\begin{gathered}
P_{n}\left(\eta ; \boldsymbol{\lambda}^{\prime}\right) \times(\cdots)+\partial_{\eta} P_{n}\left(\eta ; \boldsymbol{\lambda}^{\prime}\right) \times(\cdots)+P_{n-1}\left(\eta ; \boldsymbol{\lambda}^{\prime}+\boldsymbol{\delta}\right) \times(\cdots) \\
+\partial_{\eta} P_{n-1}\left(\eta ; \boldsymbol{\lambda}^{\prime}+\boldsymbol{\delta}\right) \times(\cdots)+\partial_{\eta}^{2} P_{n-1}\left(\eta ; \boldsymbol{\lambda}^{\prime}+\boldsymbol{\delta}\right) \times(\cdots),
\end{gathered}
$$

where $\boldsymbol{\lambda}^{\prime}=\boldsymbol{\lambda}+\ell \boldsymbol{\delta}+\tilde{\boldsymbol{\delta}}$. The above expression can be reduced to $P_{n-1}\left(\eta ; \boldsymbol{\lambda}^{\prime}+\boldsymbol{\delta}\right) \times X(\eta)+$ $\partial_{\eta} P_{n-1}\left(\eta ; \boldsymbol{\lambda}^{\prime}+\boldsymbol{\delta}\right) \times c_{2}(\eta) Y(\eta)$, by using $(2.23),(2.24),(2.17)$ and the relations $\left(c_{1}(\eta ; \boldsymbol{\lambda}+\boldsymbol{\delta}+\tilde{\boldsymbol{\delta}})-\right.$ $\left.c_{1}(\eta ; \boldsymbol{\lambda})\right) d_{2}(\eta)=2 c_{2}(\eta) \partial_{\eta} d_{2}(\eta)$ with $\mathcal{E}_{n}(\boldsymbol{\lambda})=f_{n}(\boldsymbol{\lambda}) b_{n-1}(\boldsymbol{\lambda})$. Up to an overall normalization, this $Y(\eta)$ is just the r.h.s. of (B.1), so it vanishes. Hence the backward shift relation is equivalent to $X(\eta)=0$, namely,

$$
\begin{aligned}
0= & c_{1}\left(\eta, \boldsymbol{\lambda}^{\prime}\right) d_{1}(\boldsymbol{\lambda}) \xi_{\ell}(\eta ; \boldsymbol{\lambda}+\boldsymbol{\delta})^{2}-c_{1}(\eta, \boldsymbol{\lambda}+\ell \boldsymbol{\delta}) d_{1}(\boldsymbol{\lambda}+\boldsymbol{\delta}) \xi_{\ell}(\eta ; \boldsymbol{\lambda}) \xi_{\ell}(\eta ; \boldsymbol{\lambda}+2 \boldsymbol{\delta}) \\
& -c_{2}(\eta) d_{1}(\boldsymbol{\lambda}+\boldsymbol{\delta})\left(\xi_{\ell}(\eta ; \boldsymbol{\lambda}) \partial_{\eta} \xi_{\ell}(\eta ; \boldsymbol{\lambda}+2 \boldsymbol{\delta})-\partial_{\eta} \xi_{\ell}(\eta ; \boldsymbol{\lambda}) \xi_{\ell}(\eta ; \boldsymbol{\lambda}+2 \boldsymbol{\delta})\right) \\
& +\frac{1}{4} \mathcal{E}_{1}\left(\boldsymbol{\lambda}^{\prime}\right) d_{2}(\eta) \xi_{\ell}(\eta ; \boldsymbol{\lambda}) \xi_{\ell}(\eta ; \boldsymbol{\lambda}+\boldsymbol{\delta}) .
\end{aligned}
$$

This is a polynomial identity of degree $2 \ell+1$ and it is quadratic in the Laguerre/Jacobi polynomials. With the help of (2.20) and (2.18), it is elementary to show that the r.h.s. of (B.2) becomes $\xi_{\ell}(\eta ; \boldsymbol{\lambda}+\boldsymbol{\delta}) d_{1}(\boldsymbol{\lambda}) d_{2}(\eta) \times(\cdots)$. Up to an overall normalization, this $(\cdots)$ part is just (l.h.s. of $(2.21))$ - (r.h.s. of $(2.21)$ ), so it vanishes. This concludes the proof of the backward shift relation. 


\section{Proof of the integration formula}

Here we present a simple proof for the integration formulas (7.1) or (7.2). We evaluate

$$
\begin{aligned}
& \left\langle\Xi_{\ell, \boldsymbol{\lambda}}[p(\eta)], \Xi_{\ell, \boldsymbol{\lambda}}[q(\eta)]\right\rangle_{\ell, \boldsymbol{\lambda}}=\int\left(d_{1}(\boldsymbol{\lambda}) \xi_{\ell}(\eta ; \boldsymbol{\lambda}+\boldsymbol{\delta}) p(\eta)-d_{2}(\eta) \xi_{\ell}(\eta ; \boldsymbol{\lambda}) \partial_{\eta} p(\eta)\right) \\
& \quad \times\left(d_{1}(\boldsymbol{\lambda}) \xi_{\ell}(\eta ; \boldsymbol{\lambda}+\boldsymbol{\delta}) q(\eta)-d_{2}(\eta) \xi_{\ell}(\eta ; \boldsymbol{\lambda}) \partial_{\eta} q(\eta)\right) \frac{W(\eta ; \boldsymbol{\lambda}+\ell \boldsymbol{\delta})}{\xi_{\ell}(\eta ; \boldsymbol{\lambda})^{2}} d \eta \\
& =\int\left(d_{2}(\eta)^{2} \partial_{\eta} p(\eta) \partial_{\eta} q(\eta)+\left(\frac{d_{1}(\boldsymbol{\lambda}) \xi_{\ell}(\eta ; \boldsymbol{\lambda}+\boldsymbol{\delta})}{\xi_{\ell}(\eta ; \boldsymbol{\lambda})}\right)^{2} p(\eta) q(\eta)\right. \\
& \left.\quad-\frac{d_{1}(\boldsymbol{\lambda}) \xi_{\ell}(\eta ; \boldsymbol{\lambda}+\boldsymbol{\delta})}{\xi_{\ell}(\eta ; \boldsymbol{\lambda})} d_{2}(\eta) \partial_{\eta}(p(\eta) q(\eta))\right) W(\eta ; \boldsymbol{\lambda}+\ell \boldsymbol{\delta}) d \eta \\
& \stackrel{(\mathrm{i})}{=} \int W(\eta ; \boldsymbol{\lambda}+\ell \boldsymbol{\delta})\left(d_{2}(\eta)^{2} \partial_{\eta} p(\eta) \partial_{\eta} q(\eta)\right. \\
& \left.\quad+\left(\left(\frac{d_{1}(\boldsymbol{\lambda}) \xi_{\ell}(\eta ; \boldsymbol{\lambda}+\boldsymbol{\delta})}{\xi_{\ell}(\eta ; \boldsymbol{\lambda})}\right)^{2}-\frac{\partial_{\eta}\left(W(\eta ; \boldsymbol{\lambda}+\ell \boldsymbol{\delta}) \frac{d_{1}(\boldsymbol{\lambda}) \xi_{\ell}(\eta ; \boldsymbol{\lambda}+\boldsymbol{\delta})}{\xi_{\ell}(\eta ; \boldsymbol{\lambda})} d_{2}(\eta)\right)}{W(\eta ; \boldsymbol{\lambda}+\ell \boldsymbol{\delta})}\right) p(\eta) q(\eta)\right) d \eta \\
& \stackrel{(\mathrm{ii})}{=} \int\left(d_{1}(\boldsymbol{\lambda}) d_{3}(\boldsymbol{\lambda}+\ell \boldsymbol{\delta}, \ell) p(\eta) q(\eta) W\left(\eta ; \boldsymbol{\lambda}^{\prime}\right)+\frac{1}{4} c_{\mathcal{F}}^{2} \partial_{\eta} p(\eta) \partial_{\eta} q(\eta) W\left(\eta ; \boldsymbol{\lambda}^{\prime}+\boldsymbol{\delta}\right)\right) d \eta,
\end{aligned}
$$

where $\boldsymbol{\lambda}^{\prime}=\boldsymbol{\lambda}+\ell \boldsymbol{\delta}+\tilde{\boldsymbol{\delta}}$. In (i) we have integrated by part and in (ii) we have used

$$
\begin{aligned}
& \left(\frac{d_{1}(\boldsymbol{\lambda}) \xi_{\ell}(\eta ; \boldsymbol{\lambda}+\boldsymbol{\delta})}{\xi_{\ell}(\eta ; \boldsymbol{\lambda})}\right)^{2}-\frac{\partial_{\eta}\left(W(\eta ; \boldsymbol{\lambda}+\ell \boldsymbol{\delta}) \frac{d_{1}(\boldsymbol{\lambda}) \xi_{\ell}(\eta ; \boldsymbol{\lambda}+\boldsymbol{\delta})}{\xi_{\ell}(\eta ; \boldsymbol{\lambda})} d_{2}(\eta)\right)}{W(\eta ; \boldsymbol{\lambda}+\ell \boldsymbol{\delta})} \\
& =d_{1}(\boldsymbol{\lambda}) d_{3}(\boldsymbol{\lambda}+\ell \boldsymbol{\delta}, \ell) \frac{d_{2}(\eta)^{2}}{c_{2}(\eta)},
\end{aligned}
$$

which is shown by using (2.18) and $(2.20)$ to eliminate $\xi_{\ell}(\eta ; \boldsymbol{\lambda}+\boldsymbol{\delta})$.

\section{Hyperbolic DPT potential}

Here we provide a brief summary of the properties of the $X_{\ell}$ Jacobi polynomials related to the deformed hyperbolic DPT potential. They are of the J2 type. The $\ell=1$ case was introduced in [11] and the general $\ell$ case was studied in [1]. In contrast to the radial oscillator and the trigonometric DPT potentials, the undeformed hyperbolic DPT potential allows only a finite number of square integrable polynomials $P_{n}(\eta, \boldsymbol{\lambda}), n=0,1, \ldots, n_{B} \stackrel{\text { def }}{=}\left[\frac{1}{2}(h-g)\right]^{\prime}$, where $[x]^{\prime}$ denotes the greatest integer not equal or exceeding $x$. The situation is the same for the exceptional polynomials, $P_{\ell, n}(\eta, \boldsymbol{\lambda}), n=0,1, \ldots, n_{B}-\ell$. Except for this point, the arguments for the hyperbolic DPT are the same as the radial oscillator and the trigonometric DPT potential cases. In particular, the much simpler looking new forms of the polynomials are also equivalent to the original forms of the polynomials given in [1]. We present various data:

$$
\begin{aligned}
& \boldsymbol{\lambda} \stackrel{\text { def }}{=}(g, h), \quad h>g>0, \quad \boldsymbol{\delta} \stackrel{\text { def }}{=}(1,-1), \quad P_{n}(\eta ; g, h) \stackrel{\text { def }}{=} P_{n}^{\left(g-\frac{1}{2},-h-\frac{1}{2}\right)}(\eta), \\
& \xi_{\ell}(\eta ; g, h) \stackrel{\text { def }}{=} P_{\ell}^{\left(-g-\ell-\frac{1}{2},-h+\ell-\frac{3}{2}\right)}(\eta), \quad n \leq n_{B}, \quad \ell<n_{B}, \\
& P_{\ell, n}(\eta ; \boldsymbol{\lambda}) \quad(2.5), \quad n=0,1, \ldots, n_{B}-\ell, \\
& d_{0}(n, \boldsymbol{\lambda}) \stackrel{\text { def }}{=} n+g+\frac{1}{2}, \quad \tilde{\boldsymbol{\delta}} \stackrel{\text { def }}{=}(1,1), \quad d_{1}(\boldsymbol{\lambda}) \stackrel{\text { def }}{=} g+\frac{1}{2}, \quad d_{2}(\eta) \stackrel{\text { def }}{=} 1-\eta,
\end{aligned}
$$




$$
\begin{aligned}
& W(\eta ; \boldsymbol{\lambda}) \stackrel{\text { def }}{=} \frac{1}{2^{g-h+1}}(\eta-1)^{g-\frac{1}{2}}(\eta+1)^{-h-\frac{1}{2}}, \quad 1<\eta<\infty, \\
& h_{n}(\boldsymbol{\lambda}) \stackrel{\text { def }}{=} \frac{\Gamma\left(n+g+\frac{1}{2}\right) \Gamma(h-g-n+1)}{2 n !(h-g-2 n) \Gamma\left(h-n+\frac{1}{2}\right)}, \\
& h_{\ell, n}(g, h) \stackrel{\text { def }}{=} h_{n}(g+\ell, h-\ell) \frac{\left(n+g+\ell+\frac{1}{2}\right)\left(h-n-2 \ell+\frac{1}{2}\right)}{\left(n+g+\frac{1}{2}\right)\left(h-n-\ell+\frac{1}{2}\right)}, \\
& c_{1}(\eta, \boldsymbol{\lambda}) \stackrel{\text { def }}{=} g+h+(g-h+1) \eta, \quad c_{2}(\eta) \stackrel{\text { def }}{=} \eta^{2}-1, \\
& \tilde{c}_{1}(\eta, \boldsymbol{\lambda}, \ell) \stackrel{\text { def }}{=} h-g-2 \ell+1-(g+h) \eta \text {, } \\
& \mathcal{E}_{n}(\boldsymbol{\lambda}) \stackrel{\text { def }}{=} 4 n(h-g-n), \quad \widetilde{\mathcal{E}}_{\ell}(\boldsymbol{\lambda}) \stackrel{\text { def }}{=} 4 \ell(g+h+1-\ell), \\
& d_{3}(\boldsymbol{\lambda}, \ell) \stackrel{\text { def }}{=} h-\ell+\frac{1}{2}, \quad c_{\mathcal{F}} \stackrel{\text { def }}{=} 4, \quad f_{n}(\boldsymbol{\lambda}) \stackrel{\text { def }}{=} 2(n+g-h), \quad b_{n-1}(\boldsymbol{\lambda}) \stackrel{\text { def }}{=}-2 n, \\
& P_{\ell, n}(\eta ; \boldsymbol{\lambda})=\frac{1}{2^{n} n !} \frac{\xi_{\ell}(\eta ; \boldsymbol{\lambda})}{(\eta-1)^{g+\ell-\frac{1}{2}}(\eta+1)^{-h+\ell-\frac{1}{2}}} \prod_{k=0}^{n-1}\left(\frac{d}{d \eta}+\partial_{\eta} \log \frac{\xi_{\ell}(\eta ; \boldsymbol{\lambda}+(k+1) \boldsymbol{\delta})}{\xi_{\ell}(\eta ; \boldsymbol{\lambda}+k \boldsymbol{\delta})}\right) \\
& \times \frac{(\eta-1)^{n+g+\ell-\frac{1}{2}}(\eta+1)^{n-h+\ell-\frac{1}{2}}}{\xi_{\ell}(\eta ; \boldsymbol{\lambda}+n \boldsymbol{\delta})} \xi_{\ell}(\eta ; \boldsymbol{\lambda}+(n+1) \boldsymbol{\delta}), \\
& d_{0}\left(t \partial_{t}, \boldsymbol{\lambda}\right) G_{\ell}(t, \eta ; \boldsymbol{\lambda})=\sum_{n} t^{n} d_{0}(n, \boldsymbol{\lambda}) P_{\ell, n}(\eta ; \boldsymbol{\lambda}) \\
& =\left(\left(g+\frac{1}{2}\right) P_{\ell}^{\left(-g-\ell-\frac{3}{2},-h+\ell-\frac{1}{2}\right)}(\eta)-\frac{(1-\eta) t}{R}\left(\frac{1}{R}+\frac{g+\ell+\frac{1}{2}}{1+R-t}+\frac{h-\ell+\frac{1}{2}}{1+R+t}\right) P_{\ell}^{\left(-g-\ell-\frac{1}{2},-h+\ell-\frac{3}{2}\right)}(\eta)\right) \\
& \times G^{\left(g+\ell+\frac{1}{2}, h-\ell+\frac{1}{2}\right)}(t, \eta), \quad R \stackrel{\text { def }}{=} \sqrt{1-2 \eta t+t^{2}}, \\
& \mathcal{V}_{\ell, n} \stackrel{\text { def }}{=} \operatorname{Span}\left[\eta^{k}\left(g+\frac{1}{2}\right) \xi_{\ell}(\eta ; g+1, h-1)-k(1-\eta) \eta^{k-1} \xi_{\ell}(\eta ; g, h) ; k=0,1, \ldots, n\right] \text {, } \\
& p_{n}(\eta)=(\eta-1)^{n} \quad \text { for }(8.1) \text {. }
\end{aligned}
$$

In the expression of the generating function, the summation range is extended to infinity, $\sum_{n=0}^{n_{B}-\ell} \rightarrow$

$\sum_{n=0}^{\infty}$, formally. The forward and backward shift relations (5.1) and (5.2) in terms of the Jacobi polynomials are the same as the J2 case.

\section{E Summary: properties of the polynomials}

\section{E.1 Laguerre polynomials}

- Definition (expansion formula)

$$
L_{n}^{(\alpha)}(x)=\frac{1}{n !} \sum_{k=0}^{n} \frac{(-n)_{k}}{k !}(\alpha+k+1)_{n-k} x^{k} .
$$

- Forward and backward shift relations

$$
\begin{aligned}
& \partial_{x} L_{n}^{(\alpha)}(x)=-L_{n-1}^{(\alpha+1)}(x), \\
& x \partial_{x} L_{n-1}^{(\alpha+1)}(x)+(\alpha+1-x) L_{n-1}^{(\alpha+1)}(x)=n L_{n}^{(\alpha)}(x) .
\end{aligned}
$$

- Differential equation

$$
x \partial_{x}^{2} L_{n}^{(\alpha)}(x)+(\alpha+1-x) \partial_{x} L_{n}^{(\alpha)}(x)+n L_{n}^{(\alpha)}(x)=0 .
$$


- Three term recurrence relation

$$
x L_{n}^{(\alpha)}(x)=-(n+1) L_{n+1}^{(\alpha)}(x)+(2 n+\alpha+1) L_{n}^{(\alpha)}(x)-(n+\alpha) L_{n-1}^{(\alpha)}(x) .
$$

- Rodrigues formula

$$
L_{n}^{(\alpha)}(x)=\frac{1}{n !} \frac{1}{e^{-x} x^{\alpha}}\left(\frac{d}{d x}\right)^{n}\left(e^{-x} x^{n+\alpha}\right) .
$$

- Orthogonality $(\alpha>-1)$

$$
\int_{0}^{\infty} d x e^{-x} x^{\alpha} L_{n}^{(\alpha)}(x) L_{m}^{(\alpha)}(x)=\frac{1}{n !} \Gamma(n+\alpha+1) \delta_{n m} .
$$

- Generating functions

$$
\begin{aligned}
& G^{(\alpha)}(t, x) \stackrel{\text { def }}{=} \sum_{n=0}^{\infty} t^{n} L_{n}^{(\alpha)}(x)=\frac{e^{-\frac{t x}{1-t}}}{(1-t)^{\alpha+1}}, \\
& G_{+}^{(\alpha)}(t, x) \stackrel{\text { def }}{=} \sum_{n=0}^{\infty} t^{n} L_{n}^{(\alpha+n)}(x)=\frac{2^{\alpha} e^{-\frac{1}{4} t^{-1}(1-\sqrt{1-4 t})^{2} x}}{\sqrt{1-4 t}(1+\sqrt{1-4 t})^{\alpha}}, \\
& G_{-}^{(\alpha)}(t, x) \stackrel{\text { def }}{=} \sum_{n=0}^{\infty} t^{n} L_{n}^{(\alpha-n)}(x)=(1+t)^{\alpha} e^{-t x} .
\end{aligned}
$$

Formulas (E.9) and (E.10) can be derived by using (E.2) and (E.4).

- Identities

$$
\begin{aligned}
& L_{n}^{(\alpha)}(x)-L_{n}^{(\alpha-1)}(x)=L_{n-1}^{(\alpha)}(x), \\
& x L_{n-1}^{(\alpha+1)}(x)-\alpha L_{n-1}^{(\alpha)}(x)=-n L_{n}^{(\alpha-1)}(x),
\end{aligned}
$$

which can be verified elementarily based on (E.1).

\section{E.2 Jacobi polynomials}

- Definition (expansion formula)

$$
P_{n}^{(\alpha, \beta)}(x)=\frac{(\alpha+1)_{n}}{n !} \sum_{k=0}^{n} \frac{1}{k !} \frac{(-n)_{k}(n+\alpha+\beta+1)_{k}}{(\alpha+1)_{k}}\left(\frac{1-x}{2}\right)^{k} .
$$

- Parity

$$
P_{n}^{(\alpha, \beta)}(-x)=(-1)^{n} P_{n}^{(\beta, \alpha)}(x) .
$$

- Forward and backward shift relations

$$
\begin{aligned}
& \partial_{x} P_{n}^{(\alpha, \beta)}(x)=\frac{1}{2}(n+\alpha+\beta+1) P_{n-1}^{(\alpha+1, \beta+1)}(x), \\
& \left(1-x^{2}\right) \partial_{x} P_{n-1}^{(\alpha+1, \beta+1)}(x)+(\beta-\alpha-(\alpha+\beta+2) x) P_{n-1}^{(\alpha+1, \beta+1)}(x)=-2 n P_{n}^{(\alpha, \beta)}(x) .
\end{aligned}
$$

- Differential equation

$$
\begin{aligned}
& \left(1-x^{2}\right) \partial_{x}^{2} P_{n}^{(\alpha, \beta)}(x)+(\beta-\alpha-(\alpha+\beta+2) x) \partial_{x} P_{n}^{(\alpha, \beta)}(x) \\
& \quad+n(n+\alpha+\beta+1) P_{n}^{(\alpha, \beta)}(x)=0 .
\end{aligned}
$$


- Three term recurrence relation

$$
\begin{aligned}
x P_{n}^{(\alpha, \beta)}(x)= & \frac{2(n+1)(n+\alpha+\beta+1) P_{n+1}^{(\alpha, \beta)}(x)}{(2 n+\alpha+\beta+1)(2 n+\alpha+\beta+2)}+\frac{\left(\beta^{2}-\alpha^{2}\right) P_{n}^{(\alpha, \beta)}(x)}{(2 n+\alpha+\beta)(2 n+\alpha+\beta+2)} \\
& +\frac{2(n+\alpha)(n+\beta) P_{n-1}^{(\alpha, \beta)}(x)}{(2 n+\alpha+\beta)(2 n+\alpha+\beta+1)} .
\end{aligned}
$$

- Rodrigues formula

$$
P_{n}^{(\alpha, \beta)}(x)=\frac{(-1)^{n}}{2^{n} n !} \frac{1}{(1-x)^{\alpha}(1+x)^{\beta}}\left(\frac{d}{d x}\right)^{n}\left((1-x)^{n+\alpha}(1+x)^{n+\beta}\right) .
$$

- Orthogonality $(\alpha, \beta>-1)$

$$
\begin{aligned}
\int_{-1}^{1} d x & (1-x)^{\alpha}(1+x)^{\beta} P_{n}^{(\alpha, \beta)}(x) P_{m}^{(\alpha, \beta)}(x) \\
& =\frac{2^{\alpha+\beta+1}}{n !} \frac{\Gamma(n+\alpha+1) \Gamma(n+\beta+1)}{(2 n+\alpha+\beta+1) \Gamma(n+\alpha+\beta+1)} \delta_{n m} .
\end{aligned}
$$

- Generating function

$$
\begin{aligned}
& G^{(\alpha, \beta)}(t, x) \stackrel{\text { def }}{=} \sum_{n=0}^{\infty} t^{n} P_{n}^{(\alpha, \beta)}(x)=\frac{2^{\alpha+\beta}}{R(1+R-t)^{\alpha}(1+R+t)^{\beta}}, \\
& R \stackrel{\text { def }}{=} \sqrt{1-2 x t+t^{2}} .
\end{aligned}
$$

- Identities

$$
\begin{aligned}
& 2(n+\beta) P_{n}^{(\alpha, \beta-1)}(x)-2 \beta P_{n}^{(\alpha-1, \beta)}(x)=(n+\alpha+\beta)(1+x) P_{n-1}^{(\alpha, \beta+1)}(x), \\
& (n+\beta)(1-x) P_{n-1}^{(\alpha+1, \beta)}(x)-\alpha(1+x) P_{n-1}^{(\alpha, \beta+1)}(x)=-2 n P_{n}^{(\alpha-1, \beta)}(x) \\
& 2(\alpha+n) P_{n-1}^{(\alpha, \beta)}(x)+(\alpha+\beta+2 n)(1-x)\left(P_{n}^{(\alpha, \beta)}(x)-P_{n}^{(\alpha+1, \beta-1)}(x)\right)=2 n P_{n}^{(\alpha, \beta)}(x),(x) \\
& 2(n+\alpha) P_{n}^{(\alpha-1, \beta)}(x)-2 \alpha P_{n}^{(\alpha, \beta-1)}(x)=-(n+\alpha+\beta)(1-x) P_{n-1}^{(\alpha+1, \beta)}(x), \\
& (n+\alpha)(1+x) P_{n-1}^{(\alpha, \beta+1)}(x)-\beta(1-x) P_{n-1}^{(\alpha+1, \beta)}(x)=2 n P_{n}^{(\alpha, \beta-1)}(x), \\
& -2(\beta+n) P_{n-1}^{(\alpha, \beta)}(x)+(\alpha+\beta+2 n)(1+x)\left(P_{n}^{(\alpha, \beta)}(x)-P_{n}^{(\alpha-1, \beta+1)}(x)\right)=2 n P_{n}^{(\alpha, \beta)}(x) .(
\end{aligned}
$$

Equations (E.22)-(E.24) can be verified elementarily based on (E.13). By using (E.14), equations (E.25)-(E.27) are obtained from equations (E.22)-(E.24) respectively.

- Limit to the Laguerre polynomial

$$
\lim _{\beta \rightarrow \infty} P_{n}^{(\alpha, \pm \beta)}\left(1-\frac{2 x}{\beta}\right)=L_{n}^{(\alpha)}( \pm x) .
$$

Various formulas for the Jacobi polynomials reduce to those for the Laguerre polynomials in this limit.

\section{Acknowledgments}

This work is supported in part by the National Science Council (NSC) of the Republic of China under Grant NSC 96-2112-M-032-007-MY3 (CLH), and in part by Grants-in-Aid for Scientific Research from the Ministry of Education, Culture, Sports, Science and Technology, No.19540179 (RS). Part of the work was done during CLH's visit to the Yukawa Institute for Theoretical Physics (YITP), Kyoto University, and he would like to thank the staff and members of YITP for the hospitality extended to him. RS wishes to thank National Taiwan University and National Chiao-Tung University for the hospitality extended to him during his visits in which a part of the work was done. 


\section{References}

[1] Odake S., Sasaki R., Infinitely many shape invariant potentials and new orthogonal polynomials, Phys. Lett. B 679 (2009), 414-417, arXiv:0906.0142.

[2] Odake S., Sasaki R., Another set of infinitely many exceptional $\left(X_{\ell}\right)$ Laguerre polynomials, Phys. Lett. B 684 (2010), 173-176, arXiv:0911.3442.

[3] Infeld L., Hull T.E., The factorization method, Rev. Modern Phys. 23 (1951), 21-68.

[4] Cooper F., Khare A., Sukhatme U., Supersymmetry and quantum mechanics, Phys. Rep. 251 (1995), 267385, hep-th/9405029.

[5] Darboux G., Sur une proposition relative aux équations linéaires, C.R. Acad. Paris 94 (1882), 1456-1459. Darboux G., Leçons sur la théorie des surfaces, Vol. 2, 2nd ed., Gauthier-Villars, Paris, 1915, 210-215.

Pöschl G., Teller E., Bemerkungen zur Quantenmechanik des anharmonischen Oszillators, Z. Phys. 83 (1933), 143-151.

[6] Bochner S., Über Sturm-Liouvillesche Polynomsysteme, Math. Z. 29 (1929), 730-736.

[7] Gómez-Ullate D., Kamran N., Milson R., An extension of Bochner's problem: exceptional invariant subspaces, J. Approx. Theory 162 (2010), 987-1006, arXiv:0805.3376.

Gómez-Ullate D., Kamran N., Milson R., An extended class of orthogonal polynomials defined by a SturmLiouville problem, J. Math. Anal. Appl. 359 (2009), 352-367, arXiv:0807.3939.

[8] Gómez-Ullate D., Kamran N., Milson R., Supersymmetry and algebraic Darboux transformations, J. Phys. A: Math. Gen. 37 (2004), 10065-10078, nlin.SI/0402052.

Gómez-Ullate D., Kamran N., Milson R., Quasi-exact solvability and the direct approach to invariant subspaces, J. Phys. A: Math. Gen. 38 (2005), 2005-2019, nlin.SI/0401030.

[9] Gendenshtein L.E., Derivation of exact spectra of the Schrödinger equation by means of supersymmetry, JETP Lett. 38 (1983), 356-359.

[10] Quesne C., Exceptional orthogonal polynomials, exactly solvable potentials and supersymmetry, J. Phys. A: Math. Theor. 41 (2008), 392001, 6 pages, arXiv:0807.4087.

[11] Bagchi B., Quesne C., Roychoudhury R., Isospectrality of conventional and new extended potentials, secondorder supersymmetry and role of PT symmetry, Pramana J. Phys. 73 (2009), 337-347, arXiv:0812.1488.

[12] Crum M.M., Associated Sturm-Liouville systems, Quart. J. Math. Oxford Ser. (2) 6 (1955), 121-127, physics/9908019.

[13] Quesne C., Solvable rational potentials and exceptional orthogonal polynomials in supersymmetric quantum mechanics, SIGMA 5 (2009), 084, 24 pages, arXiv:0906.2331.

[14] Tanaka T., $\mathcal{N}$-fold supersymmetry and quasi-solvability associated with $X_{2}$-Laguerre polynomials, J. Math. Phys. 51 (2010), 032101, 20 pages, arXiv:0910.0328.

[15] Odake S., Sasaki R., Infinitely many shape invariant potentials and cubic identities of the Laguerre and Jacobi polynomials, J. Math. Phys. 51 (2010), 053513, 9 pages, arXiv:0911.1585.

[16] Odake S., Sasaki R., Infinitely many shape invariant discrete quantum mechanical systems and new exceptional orthogonal polynomials related to the Wilson and Askey-Wilson polynomials, Phys. Lett. B 682 (2009), 130-136, arXiv:0909.3668.

[17] Gómez-Ullate D., Kamran N., Milson R., Exceptional orthogonal polynomials and the Darboux transformation, J. Phys. A: Math. Theor. 43 (2010), 434016, 16 pages, arXiv:1002.2666.

[18] Sasaki R., Tsujimoto S., Zhedanov A., Exceptional Laguerre and Jacobi polynomials and the corresponding potentials through Darboux-Crum transformations, J. Phys. A: Math. Theor. 43 (2010), 315204, 20 pages, arXiv:1004.4711.

[19] Andrews G.E., Askey R., Roy R., Special functions, Encyclopedia of Mathematics and its Applications, Vol. 71, Cambridge University Press, Cambridge, 1999.

[20] Heine H.E., Theorie der Kugelfunctionen und der verwandten Functionen, Berlin, 1878.

Stieltjes T.J., Sur certains polynômes qui vérifient une équation différentielle linéaire du second ordre et sur la théorie des fonctions de Lamé, Acta Math. 6 (1885), 321-326. 